TRANSACTIONS OF THE

AMERICAN MATHEMATICAL SOCIETY

Volume 357, Number 11, Pages 4379-4412

S 0002-9947(05)03947-4

Article electronically published on June 9, 2005

\title{
ON THE POWER SERIES COEFFICIENTS OF CERTAIN QUOTIENTS OF EISENSTEIN SERIES
}

\author{
BRUCE C. BERNDT AND PAUL R. BIALEK
}

\begin{abstract}
In their last joint paper, Hardy and Ramanujan examined the coefficients of modular forms with a simple pole in a fundamental region. In particular, they focused on the reciprocal of the Eisenstein series $E_{6}(\tau)$. In letters written to Hardy from nursing homes, Ramanujan stated without proof several more results of this sort. The purpose of this paper is to prove most of these claims.
\end{abstract}

\section{INTRODUCTION}

In their epic paper [9], 18, pp. 276-309], G. H. Hardy and S. Ramanujan found an asymptotic formula for the partition function $p(n)$, which arises from the power series coefficients of the reciprocal of the Dedekind eta-function, a modular form of negative dimension (positive weight). As they indicated near the end of their paper, their methods also apply to several analogues of the partition function generated by modular forms of negative weight that are analytic in the upper half-plane. In their last published paper [10], 18, pp. 310-321], they considered a similar problem for the coefficients of modular forms of negative weight having a simple pole in the fundamental region, and, in particular, they applied their theorem to find interesting series representations for the coefficients of the reciprocal of the Eisenstein series $E_{6}(\tau)$. Although there are some similarities in the methods of these papers, the principal ideas in [10] are quite different from those in [9. In [9, Hardy and Ramanujan introduced their famous circle method, and since that time the ideas in this paper have had an enormous impact in additive analytic number theory. Although their paper [10] has not had as much influence, the ideas in [10] have been extended by J. Lehner [12, H. Petersson [13, [14, [15, H. Poincaré [16, and H. S. Zuckerman [22]. Additional comments on [10] can be found in the third edition of [18, p. 387].

While confined to nursing homes and sanitariums during his last two years in England, Ramanujan wrote several letters to Hardy about the coefficients in the power series expansions of certain quotients of Eisenstein series. A few pages in his lost notebook are also devoted to this topic. All of this material can be found in [19, pp. 97-126], and the letters with commentary can be found in the book

Received by the editors September 30, 2000 and, in revised form, June 1, 2003.

2000 Mathematics Subject Classification. Primary 11F30, 11F27, 33E05.

Key words and phrases. Eisenstein series, modular forms, formulas for power series coefficients, Ramanujan's letters to Hardy.

The first author's research was partially supported by grant MDA904-00-1-0015 from the National Security Agency.

(C)2005 American Mathematical Society Reverts to public domain 28 years from publication 
by Berndt and R. A. Rankin [6] pp. 175-191]. In these letters and in the lost notebook, Ramanujan claims formulas for the coefficients of several quotients of Eisenstein series not examined by Hardy and him in [10]. In fact, for some of these quotients, the main theorem of [10] needs to be modified or improved. Ramanujan obviously wanted another example to be included in their paper [10], for in his letter of 28 June 1918 [6. pp. 182-183], he wrote, "I am sending you the analogous results in case of $g_{2}$. Please mention them in the paper without proof. After all we have got only two neat examples to offer, viz. $g_{2}$ and $g_{3}$. So please don't omit the results." This letter was evidently written after galley proofs for [10] were printed, for Ramanujan's request went unheeded. The functions $g_{2}$ and $g_{3}$ are the familiar invariants in the theory of elliptic functions and are constant multiples of the Eisenstein series $E_{4}(\tau)$ and $E_{6}(\tau)$, respectively. The letter was also evidently written before Ramanujan obtained further examples.

In this paper, we establish the formulas for the coefficients of those quotients of Eisenstein series found in [19, pp. 102-104, 117]. In Ramanujan's notation, the three relevant Eisenstein series are defined, for $|q|<1$, by

$$
\begin{aligned}
& P(q):=1-24 \sum_{k=1}^{\infty} \frac{k q^{k}}{1-q^{k}}, \\
& Q(q):=1+240 \sum_{k=1}^{\infty} \frac{k^{3} q^{k}}{1-q^{k}},
\end{aligned}
$$

and

$$
R(q):=1-504 \sum_{k=1}^{\infty} \frac{k^{5} q^{k}}{1-q^{k}}
$$

In more contemporary notation, the Eisenstein series $E_{2 j}(\tau)$ is defined for $j>1$ and $\operatorname{Im} \tau>0$ by

$$
E_{2 j}(\tau):=\frac{1}{2} \sum_{\substack{m_{1}, m_{2} \in \mathbf{Z} \\\left(m_{1}, m_{2}\right)=1}}\left(m_{1} \tau+m_{2}\right)^{-2 j}=1+\frac{(2 \pi)^{2 j}(-1)^{j}}{\zeta(2 j)(2 j-1) !} \sum_{k=1}^{\infty} \frac{k^{2 j-1} e^{2 \pi i k \tau}}{1-e^{2 \pi i k \tau}}
$$

where $\zeta(s)$ denotes the Riemann zeta-function. Thus, for $q=\exp (2 \pi i \tau), E_{4}(\tau)=$ $Q(q)$ and $E_{6}(\tau)=R(q)$, which have weights 4 and 6 , respectively [21, p. 50]. Since (1.4) does not converge for $j=1$, the Eisenstein series $E_{2}(\tau)$ must be defined differently. First let

$$
E_{2}^{*}(\tau):=P(q), \quad q=e^{2 \pi i \tau} .
$$

Then $E_{2}(\tau)$ is defined by

$$
E_{2}(\tau):=E_{2}^{*}(\tau)-\frac{3}{\pi \operatorname{Im} \tau} .
$$

Then $E_{2}(\tau)$ satisfies the functional equation of a modular form of weight 2 [21, pp. 67-68].

As indicated above, in [10], Hardy and Ramanujan obtained representations for the coefficients of $1 / R(q)$ as infinite series. In this paper, we establish Ramanujan's similar claims for the series

$$
\frac{1}{Q(q)}, \quad \frac{Q(q)}{R(q)}, \quad \frac{P(q)}{R(q)}, \quad \frac{P^{2}(q)}{R(q)}, \quad \text { and } \quad \frac{P(q)}{Q(q)} .
$$


In [19, pp. 97-101], Ramanujan established upper and lower bounds for the number of terms in the aforementioned representations for the coefficients of $1 / R(q)$ needed to explicitly determine the actual (integral) coefficients. Although some details are given in [19], expanded arguments can be found in Bialek's thesis [7. We do not have plans to publish these details in a journal. However, they will be published in the forthcoming book by G. E. Andrews and Berndt [1. Other results claimed by Ramanujan on pages $97-126$ of 19 are examined in a paper by the authors and A. J. Yee [5].

Almost all of the results in this paper can be found in Chapter 2 of Bialek's doctoral dissertation 7].

We complete the Introduction by setting notation. The set of rational integers is denoted by $\mathbf{Z}$, with $\mathbf{Z}^{+}$denoting the set of positive integers. The upper half-plane $\mathcal{H}$ is defined by

$$
\mathcal{H}=\{\tau: \operatorname{Im} \tau>0\}
$$

The residue of a function $f$ at a pole $z_{0}$ is denoted by $\operatorname{Res}\left(f, z_{0}\right)$.

We let $P_{1}$ denote the fundamental region

$$
P_{1}=\left\{\tau: \operatorname{Im} \tau>0, \quad \frac{1}{2}<\operatorname{Re} \tau<\frac{1}{2}, \quad|\tau|>1\right\} .
$$

Further fundamental regions are $P_{2}$, the region in $\mathcal{H}$ bounded by the three circles $|\tau|=1,|\tau+1|=1$, and $|\tau-1|=1 ; P_{3}$, the region in $\mathcal{H}$ bounded by the circles $|\tau-1|=1$ and $\left|\tau-\frac{1}{3}\right|=\frac{1}{3}$ and the line $\operatorname{Re} \tau=\frac{1}{2}$; and $P_{4}$, the region in $\mathcal{H}$ bounded by the circle $|\tau+1|=1$ and the lines $\operatorname{Re} \tau=-\frac{3}{2}$ and $\operatorname{Re} \tau=-\frac{1}{2}$. However, the fundamental region most important for us is the fundamental region $P$ in $\mathcal{H}$ bounded by the circles $|\tau+1|=1$ and $|\tau|=1$ and the lines $\operatorname{Re} \tau=-\frac{1}{2}-\epsilon$ and $\operatorname{Re} \tau=\frac{1}{2}-\epsilon$, where $0<\epsilon<1$.

\section{THE KEY THEOREM}

The principal tool in proving Ramanujan's formulas is the following theorem, which is essentially due to Hardy and Ramanujan [10, [18, pp. 312, 316]. However, we need to modify it slightly (by refining the estimate of their integral). In particular, in two of our applications, we examine $1 / E_{4}(\tau)$, which has a pole at a point on the boundary of $P_{1}$, and so we need to work on the fundamental region $P$ instead of $P_{1}$, both defined at the end of Section 1. For the convenience of readers who may be reading our paper while consulting or comparing it with that of Hardy and Ramanujan [10], we have adhered to the notation of [10]. In particular, they set $q=e^{\pi i \tau}$ instead of the more customary $q=e^{2 \pi i \tau}$ and therefore consider functions with arguments $q^{2}$.

Theorem 2.1. Suppose that $f(q)=f\left(e^{\pi i \tau}\right)=\varphi(\tau)$ is analytic for $q=0$, is meromorphic in the unit circle, and satisfies the functional equation

$$
\varphi(\tau)=\varphi\left(\frac{a \tau+b}{c \tau+d}\right)(c \tau+d)^{n}
$$

where $a, b, c, d \in \mathbf{Z}$; $a d-b c=1$; and $n \in \mathbf{Z}^{+}$. If $\varphi(\tau)$ has only one pole in the fundamental region $P_{1}$, a simple pole at $\tau=\alpha$ with residue $A$, then

$$
f(q)=-2 \pi i A \sum \frac{1}{(c \alpha+d)^{n+2}} \frac{1}{1-(q / \underline{q})^{2}}, \quad|q|<1,
$$


and

$$
0=-2 \pi i A \sum \frac{1}{(c \alpha+d)^{n+2}} \frac{1}{1-(q / \underline{q})^{2}}, \quad|q|>1,
$$

where

$$
\underline{q}=\exp \left(\left(\frac{a \alpha+b}{c \alpha+d}\right) \pi i\right),
$$

and the summation runs over all pairs of coprime integers $(c, d)$ which yield distinct values for the set $\{\underline{q},-\underline{q}\}$, and $a$ and $b$ are any integral solutions of

$$
a d-b c=1 .
$$

Proof. Consider the integral

$$
\frac{1}{2 \pi i} \int_{H_{m}} \frac{f(z)}{z-q} d z
$$

where $f$ is a function which satisfies the conditions specified in the theorem, $H_{m}$ is a simple closed contour which is very close to (to be made more precise in the sequel) and inside (or perhaps touching) the unit circle, and $q$ is fixed and inside $H_{m}$. By Cauchy's Theorem, if $|q|<1$,

$$
\frac{1}{2 \pi i} \int_{H_{m}} \frac{f(z)}{z-q} d z=f(q)+\sum_{m} \operatorname{Res},
$$

where $\sum_{m}$ Res is the sum of the residues of $f(z) /(z-q)$ at the poles of $f$ which are inside $H_{m}$. If we can show that the integral tends to zero as $m \rightarrow \infty$, then it will follow that $f(q)=-\sum$ Res, where the sum is over all residues of poles on the interior of $|z|=1$.

First, we construct a contour $H_{m}$ which will allow us to easily evaluate the integral. Our contour $H_{m}$ is based on Farey fractions of order $m$. For basic properties of the Farey functions of order $m$, which we denote by $F_{m}$, see, for example, [11. pp. 297-300]. For instance, if $h^{\prime} / k^{\prime}<h / k$ are two adjacent Farey fractions in $F_{m}$, then

$$
h k^{\prime}-h^{\prime} k=1
$$

also,

$$
k^{\prime}+k>m .
$$

We now construct the desired contour. Suppose $h^{\prime} / k^{\prime}<h / k$ are adjacent Farey fractions in $F_{m}$. Construct the two semicircles in $\mathcal{H}$ which have the segments

$$
\left(\frac{h^{\prime}}{k^{\prime}}, \frac{(1+2 \epsilon) h^{\prime}+2 h}{(1+2 \epsilon) k^{\prime}+2 k}\right) \quad \text { and } \quad\left(\frac{(1-2 \epsilon) h+2 h^{\prime}}{(1-2 \epsilon) k+2 k^{\prime}}, \frac{h}{k}\right)
$$

on the real axis as their diameters, where $\epsilon>0$. The inequalities

$$
\frac{h^{\prime}}{k^{\prime}}<\frac{h+2 h^{\prime}}{k+2 k^{\prime}}<\frac{h^{\prime}+2 h}{k^{\prime}+2 k}<\frac{h}{k},
$$

which follow from (2.7), imply that the circles intersect for $\epsilon$ sufficiently small.

Let us say that $N$ is their intersection point in the upper half-plane, $\omega_{L}$ is the arc from $h^{\prime} / k^{\prime}$ to $N, \omega_{R}$ is the $\operatorname{arc}$ from $N$ to $h / k$, and $\omega$ is the union of $\omega_{L}$ and $\omega_{R}$. 
Repeat the process for each adjacent pair of Farey fractions between 0 and 1. Thus we obtain a path from 0 to 1 . Construct the mirror image of this path on the interval $[-1,0]$, and call the entire contour (from -1 to 1) $\Omega_{m}$.

If we regard $\Omega_{m}$ as being in the $\tau$-plane, then the corresponding path in the $q$-plane, where $q=e^{\pi i \tau}$, is a simple closed contour which starts and ends at -1 and does not go outside the unit circle. This is our desired contour $H_{m}$. (Eventually, we shall let $m$ go to $\infty$ so that $H_{m}$ approaches the unit circle, as will be shown later.)

Now we show that each segment $\omega_{L}$ of the path $\Omega_{m}$ is the pre-image of part of the left-hand boundary of the fundamental region $P$ in $\mathcal{H}$ bounded by the circles $|\tau+1|=1$ and $|\tau|=1$ and the lines $\operatorname{Re} \tau=-\frac{1}{2}-\epsilon$ and $\operatorname{Re} \tau=\frac{1}{2}-\epsilon$ under some modular transformation, and that each segment $\omega_{R}$ is the pre-image of the righthand boundary of $P$ and a short line segment under some modular transformation. Later we use these properties to estimate $f$ on the contour $\Omega_{m}$.

If $h^{\prime} / k^{\prime}<h / k$ are adjacent Farey fractions, then

$$
T_{1}(\tau):=\frac{k^{\prime} \tau-h^{\prime}}{-k \tau+h} \quad \text { and } \quad T_{2}(\tau):=\frac{k \tau-h}{k^{\prime} \tau-h^{\prime}}
$$

are modular transformations because $h k^{\prime}-h^{\prime} k=1$. These are the modular transformations to which we referred in the previous paragraph.

We first examine the transformation $T_{1}$. Note that under $T_{1}$, the pre-images of the points $i \infty, \frac{1}{2}-\epsilon,-\frac{1}{2}-\epsilon, 1$, and -1 are

$$
\frac{h}{k}, \quad \frac{(1-2 \epsilon) h+2 h^{\prime}}{(1-2 \epsilon) k+2 k^{\prime}}, \quad \frac{(1+2 \epsilon) h-2 h^{\prime}}{(1+2 \epsilon) k-2 k^{\prime}}, \quad \frac{h+h^{\prime}}{k+k^{\prime}}, \quad \text { and } \quad \frac{h-h^{\prime}}{k-k^{\prime}},
$$

respectively. Recall that modular transformations map the family of circles and straight lines onto itself, and note from the definition of $T_{1}$ that $T_{1}(\bar{\tau})=\overline{T_{1}(\tau)}$.

These imply that the pre-images of the half-line $\operatorname{Re} \tau=\frac{1}{2}-\epsilon, \operatorname{Im} \tau \geq 0$, the half-line $\operatorname{Re} \tau=-\frac{1}{2}-\epsilon, \operatorname{Im} \tau \geq 0$, and the upper half of the unit circle are the semicircles in the upper half-plane $\mathcal{H}$ which have the segments on the real axis

$$
\left(\frac{(1-2 \epsilon) h+2 h^{\prime}}{(1-2 \epsilon) k+2 k^{\prime}}, \frac{h}{k}\right), \quad\left(\frac{h}{k}, \frac{(1+2 \epsilon) h-2 h^{\prime}}{(1+2 \epsilon) k-2 k^{\prime}}\right), \quad \text { and } \quad\left(\frac{h^{\prime}-h}{k^{\prime}-k}, \frac{h^{\prime}+h}{k^{\prime}+k}\right)
$$

as their diameters, respectively. Unless otherwise stated, the semicircles in this paper are assumed to be in $\mathcal{H}$ with their diameters on the real axis.

Similarly, under the transformation $T_{2}$, the pre-images are the semicircles which have the segments

$$
\left(\frac{h^{\prime}}{k^{\prime}}, \frac{(1-2 \epsilon) h^{\prime}-2 h}{(1-2 \epsilon) k^{\prime}-2 k}\right), \quad\left(\frac{h^{\prime}}{k^{\prime}}, \frac{(1+2 \epsilon) h^{\prime}+2 h}{(1+2 \epsilon) k^{\prime}+2 k}\right), \quad \text { and } \quad\left(\frac{h^{\prime}-h}{k^{\prime}-k}, \frac{h^{\prime}+h}{k^{\prime}+k}\right)
$$

as their diameters, respectively. Also, the pre-image of the semicircle centered at -1 with radius 1 is the semicircle with the segment

$$
\left(\frac{h+2 h^{\prime}}{k+2 k^{\prime}}, \frac{h}{k}\right)
$$

as its diameter.

From (2.9) and (2.14), we see that $T_{2}$ maps $\omega_{L}$ onto the half-line $\operatorname{Re} \tau=-\frac{1}{2}-$ $\epsilon, \operatorname{Im} \tau \geq 0$. Also, by (2.9) and (2.13), we see that $T_{1}$ maps $\omega_{R}$ onto the half-line $\operatorname{Re} \tau=\frac{1}{2}-\epsilon, \operatorname{Im} \tau \geq 0$. 
Under $T_{1}$, the image of the left semicircle is the semicircle with the segment $(0,2 /(1+2 \epsilon))$ as its diameter, while the image of the right semicircle is the halfline $\operatorname{Re} \tau=\frac{1}{2}-\epsilon, \operatorname{Im} \tau \geq 0$. The images intersect at the point

$$
\tau_{1}:=\left(\frac{1}{2}-\epsilon\right)+i \sqrt{\frac{\frac{3}{4}-\frac{3}{2} \epsilon+\epsilon^{2}-2 \epsilon^{3}}{1+2 \epsilon}},
$$

so $\tau_{1}$ must be the image of $N$. Thus the image of $\omega_{R}$ is the half-line

$$
\operatorname{Re} \tau=\frac{1}{2}-\epsilon, \quad \operatorname{Im} \tau \geq \sqrt{\frac{\frac{3}{4}-\frac{3}{2} \epsilon+\epsilon^{2}-2 \epsilon^{3}}{1+2 \epsilon}} .
$$

Similarly, under $T_{2}$, the images of the semicircles are the half-line $\operatorname{Re} \tau=-\frac{1}{2}-$ $\epsilon, \operatorname{Im} \tau \geq 0$, and the semicircle with the segment $(-2 /(1-2 \epsilon), 0)$ as its diameter. These images intersect at

$$
\tau_{2}:=\left(-\frac{1}{2}-\epsilon\right)+i \sqrt{\frac{\frac{3}{4}+\frac{3}{2} \epsilon+\epsilon^{2}+2 \epsilon^{3}}{1-2 \epsilon}},
$$

the image of $N$. Thus the image of $w_{L}$ is the half-line

$$
\operatorname{Re} \tau=-\frac{1}{2}-\epsilon, \quad \operatorname{Im} \tau \geq \sqrt{\frac{\frac{3}{4}+\frac{3}{2} \epsilon+\epsilon^{2}+2 \epsilon^{3}}{1-2 \epsilon}} .
$$

The intersection of the line $\operatorname{Re} \tau=\frac{1}{2}-\epsilon$ and the upper half of the unit circle is the point

$$
\tau_{3}:=\left(\frac{1}{2}-\epsilon\right)+i \sqrt{\frac{3}{4}+\epsilon-\epsilon^{2}}
$$

which is the lowermost point on the right-hand boundary of $P$. Similarly, the lowermost point on the left-hand boundary of $P$ is

$$
\tau_{4}:=\left(-\frac{1}{2}-\epsilon\right)+i \sqrt{\frac{3}{4}+\epsilon-\epsilon^{2}} .
$$

Note that for $\epsilon>0$,

$$
\sqrt{\frac{\frac{3}{4}-\frac{3}{2} \epsilon+\epsilon^{2}-2 \epsilon^{3}}{1+2 \epsilon}}<\sqrt{\frac{3}{4}+\epsilon-\epsilon^{2}},
$$

because

$$
\frac{3}{4}-\frac{3}{2} \epsilon+\epsilon^{2}-2 \epsilon^{3}<\left(\frac{3}{4}+\epsilon-\epsilon^{2}\right)(1+2 \epsilon)=\frac{3}{4}+\frac{5}{2} \epsilon+\epsilon^{2}-2 \epsilon^{3} .
$$

Similarly,

$$
\sqrt{\frac{3}{4}+\epsilon-\epsilon^{2}}<\sqrt{\frac{\frac{3}{4}+\frac{3}{2} \epsilon+\epsilon^{2}+2 \epsilon^{3}}{1-2 \epsilon}} .
$$

By (2.17) and (2.18), inequalities (2.20) and (2.21) imply that $T_{2}$ maps $\omega_{L}$ onto part of the left-hand boundary of $P$, while the transformation $T_{1}$ maps $\omega_{R}$ onto the right-hand boundary of $P$ and a line segment below it. We denote by $\ell_{1}$ the line segment which has $\tau_{1}$ and $\tau_{3}$ as its end points. Note that, by (2.16) and (2.19), the length of $\ell_{1}$ tends to 0 as $\epsilon$ tends to 0 .

Also, if $\omega_{L}, \omega_{R}$, and $\omega$ are curves in the $\tau$-plane, then let $\mathcal{C}_{L}, \mathcal{C}_{R}$, and $\mathcal{C}$ be the corresponding arcs in the $q$-plane where $q=e^{\pi i \tau}$. 
Next we obtain an estimate for the integral of $f(z) /(z-q)$ over $\mathcal{C}_{R}$, one segment of $H_{m}$. We then use this to obtain an estimate of the integral over all of $H_{m}$. We begin by finding an upper bound for $|f|$ on $\mathcal{C}_{R}$ (which is the same as finding an upper bound for $|\varphi|$ on $\left.\omega_{R}\right)$.

Recall that $f(q)=f\left(e^{\pi i \tau}\right)=\varphi(\tau)$ is analytic at $q=0$. So $\lim _{q \rightarrow 0} f(q)$ exists and $\lim _{\tau \rightarrow i \infty} \varphi(\tau)$ exists. Recall also that $\varphi$ has only one pole in each fundamental region. So $\varphi$ has only one pole in $P_{1}$ and one pole in $P_{4}$. Thus we see that by our choice of $\epsilon$ we can avoid having a pole on the left- or right-hand boundaries of $P$. Since $\lim _{\tau \rightarrow i \infty} \varphi(\tau)$ exists, we know that $|\varphi(\tau)|$ is bounded on the right- and left-hand boundaries of $P$.

Now consider the line segment $\ell_{1}$. If $\epsilon$ is sufficiently small, then $\ell_{1}$ is in at most two fundamental regions, $P_{2}$ and $P_{3}$. Since each of these regions has only one pole of $\varphi$, we can avoid having a pole on $\ell_{1}$ by choosing $\epsilon$ carefully. Thus, $|\varphi(\tau)|$ is bounded on $\ell_{1}$ as well. So we can say that

$$
|\varphi| \leq M
$$

on $\ell_{1}$ and the right- and left-hand boundaries of $P$, where $M$ is some absolute constant. Note that $M$ is independent of $m$, where $m$ is the order of $F_{m}$.

By the functional equation (2.1),

$$
|\varphi(\tau)|=\left|\varphi\left(\frac{k^{\prime} \tau-h^{\prime}}{-k \tau+h}\right)\right||-k \tau+h|^{n} .
$$

If $\tau$ is on $w_{R}$, then

$$
|\varphi(\tau)|<M|-k \tau+h|^{n}=M k^{n}|\tau-h / k|^{n},
$$

because $T_{1}$ maps $\omega_{R}$ onto $\ell_{1}$ and the right-hand boundary of $P$.

For $\tau$ on $\omega_{R_{1}}$, the quantity $|\tau-h / k|$ is maximized when $\tau=N$. We need to estimate $|N-h / k|$. By (2.16), $N$ is the pre-image of $\tau_{1}$ under the transformation $T_{1}$, and so

$$
N=T_{1}^{-1}\left(\tau_{1}\right)=\frac{h \tau_{1}+h^{\prime}}{k \tau_{1}+k^{\prime}} .
$$

Thus, by (2.7) and (2.16),

$$
\begin{aligned}
\left|N-\frac{h}{k}\right| & =\frac{1}{\left|k k^{\prime}+k^{2} \tau_{1}\right|} \\
& =\frac{1}{\left|k k^{\prime}+k^{2}\left(\frac{1}{2}-\epsilon\right)+i k^{2} \sqrt{\frac{\frac{3}{4}-\frac{3}{2} \epsilon+\epsilon^{2}-2 \epsilon^{3}}{1+2 \epsilon} \mid}\right|} \\
& =\frac{1}{\sqrt{\left(k k^{\prime}+k^{2}\left(\frac{1}{2}-\epsilon\right)\right)^{2}+k^{4}\left(\frac{\frac{3}{4}-\frac{3}{2} \epsilon+\epsilon^{2}-2 \epsilon^{3}}{1+2 \epsilon}\right)}} \\
& <\frac{1}{\sqrt{\left(\frac{1}{2} k k^{\prime}+\frac{1}{4} k^{2}\right)^{2}+\frac{3}{16} k^{4}}} \\
& =\frac{2}{k \sqrt{k^{2}+k k^{\prime}+k^{\prime 2}}},
\end{aligned}
$$

for $\epsilon$ sufficiently small. 
On $\omega_{R}$, by (2.24) and (2.25),

$$
|\varphi(\tau)|<M k^{n}\left(\frac{2}{k \sqrt{k^{2}+k k^{\prime}+k^{\prime 2}}}\right)^{n}=\frac{2^{n} M}{\left(k^{2}+k k^{\prime}+k^{\prime 2}\right)^{n / 2}} .
$$

Thus, we have obtained a bound for $|f|$ on $\mathcal{C}_{R}$.

If $q$ is fixed and inside $H_{m}$, then

$$
\left|\frac{f(z)}{z-q}\right|<\frac{M_{1}}{\left(k^{2}+k k^{\prime}+k^{\prime 2}\right)^{n / 2}}
$$

for $z$ on $\mathcal{C}_{R}$, where $M_{1}$ is some constant which depends on $q$ and $n$.

Now we estimate the length of $\mathcal{C}_{R}$. We first calculate the arc length of $\omega_{R}$, then make the change of variable $q=\exp (\pi i \tau)$, and lastly estimate $\mathcal{C}_{R}$. From (2.9) and (2.10), we see that the length of $\omega_{R}$ is less than

$$
\frac{1}{2} \pi\left(\frac{h}{k}-\frac{h^{\prime}}{k^{\prime}}\right)=\frac{1}{2} \pi\left(\frac{1}{k k^{\prime}}\right)
$$

by (2.7). Because

$$
\left|\frac{d q}{d \tau}\right|=\left|\pi i e^{\pi i \tau}\right|=|\pi i q| \leq \pi
$$

on this arc, the length of $\mathcal{C}_{R}$ is less than

$$
\frac{\pi^{2}}{2} \frac{1}{k k^{\prime}} \text {. }
$$

Thus, by (2.27) and (2.28),

$$
\left|\int_{\mathcal{C}_{R}} \frac{f(z)}{z-q} d z\right|<\frac{M_{2}}{k k^{\prime}\left(k^{2}+k k^{\prime}+k^{\prime 2}\right)^{n / 2}}
$$

where $M_{2}$ is some constant which depends on $q$ and $n$. Using the transformation $T_{2}$, we can obtain an identical result for the integral over $\mathcal{C}_{L}$.

So far we have examined only the portion of $\Omega_{m}$ in the right half-plane and the portion of $H_{m}$ in the upper half-plane. Because $\Omega_{m}$ is symmetric about 0 , it follows that $H_{m}$ is symmetric about the real axis. By applying the reasoning above to the interval $\left[-h / k,-h^{\prime} / k^{\prime}\right]$, we obtain identical results for the arcs in the lower half-plane which are mirror images of the $\operatorname{arcs} \mathcal{C}_{L}$ and $\mathcal{C}_{R}$ in the upper half-plane, which we have just analyzed.

We are ready to estimate the integral over $H_{m}$. Using (2.29), we obtain the inequality

$$
\left|\int_{H_{m}} \frac{f(z)}{z-q}\right|<4 M_{2} \sum_{\left(\frac{h^{\prime}}{k^{\prime}}, \frac{h}{k}\right)} \frac{1}{k k^{\prime}\left(k^{2}+k k^{\prime}+k^{\prime 2}\right)^{n / 2}},
$$

where the summation runs over all adjacent pairs of Farey fractions in $F_{m}$, and $M_{2}$ is, of course, independent of $m$. We want to show that the right-hand side tends to zero as $m$ tends to $\infty$. To that end, observe that

$$
\begin{aligned}
\sum_{\left(\frac{h^{\prime}}{k^{\prime}}, \frac{h}{k}\right)} \frac{1}{k k^{\prime}\left(k^{2}+k k^{\prime}+k^{\prime 2}\right)^{n / 2}} & <\sum_{\left(\frac{h^{\prime}}{k^{\prime}}, \frac{h}{k}\right)} \frac{1}{k k^{\prime}\left(\frac{k+k^{\prime}}{2}\right)^{n}} \\
& <\left(\frac{2}{m}\right)^{n} \sum_{\left(\frac{h^{\prime}}{k^{\prime}}, \frac{h}{k}\right)}\left(\frac{h}{k}-\frac{h^{\prime}}{k^{\prime}}\right)=\left(\frac{2}{m}\right)^{n} .
\end{aligned}
$$


Therefore, by (2.30) and (2.31),

$$
\lim _{m \rightarrow \infty} \frac{1}{2 \pi i} \int_{H_{m}} \frac{f(z)}{z-q}=0 .
$$

Note that for a typical $\operatorname{arc} \mathcal{C}$, the maximum distance from the arc to the unit circle is less than the length of $\mathcal{C}$. From our calculation of the maximum possible length of $\mathcal{C}_{R}$ in (2.28), we see that the length of $\mathcal{C}$ is less than

$$
\frac{\pi^{2}}{k k^{\prime}}<\frac{\pi^{2}}{k(m-k)} \leq \frac{\pi^{2}}{m-1}
$$

if $k<m$, and it is less than

$$
\frac{\pi^{2}}{k k^{\prime}} \leq \frac{\pi^{2}}{m}
$$

if $k=m$. So as $m$ tends to $\infty, H_{m}$ approaches the unit circle uniformly from the inside. Therefore all the poles of $f(z)$ which are inside the unit circle are eventually inside $H_{m}$. By (2.6) and (2.32)

$$
f(q)=-\sum R
$$

where $\sum R$ is the sum of the residues of $f(z) /(z-q)$ at the poles of $f$ which are inside the unit circle. We next determine these poles.

Recall that $\varphi(\tau)$ has only one pole in $P_{1}$, a simple pole at $\tau=\alpha$, with residue $A$. By the functional equation (2.1), the only poles of $\varphi(\tau)$ in the upper half-plane are at the points $\tau=(a \alpha+b) /(c \alpha+d)$, where $a, b, c, d \in \mathbf{Z}$ and $a d-b c=1$.

If $c$ and $d$ are fixed, and $(a, b)$ is one solution to $a d-b c=1$, then the complete set of solutions is $\{(a+m c, b+m d): m \in \mathbf{Z}\}$. Each of these solutions produces a distinct pole of $\varphi(\tau)$. However, this set yields only two distinct poles of $f(q)$, namely,

$$
q= \pm \exp \left(\pi i \frac{a \alpha+b}{c \alpha+d}\right)
$$

because

$$
\exp \left(\pi i \frac{(a+m c) \alpha+(b+m d)}{c \alpha+d}\right)=(-1)^{m} \exp \left(\pi i \frac{a \alpha+b}{c \alpha+d}\right)
$$

If we let $(c, d)$ range over all pairs of coprime integers, then the two expressions in (2.34) will eventually take on as its values each of the poles of $f$ inside the unit circle.

However, as we will see later when applying the theorem, it is possible that different pairs $(c, d)$ may produce the same poles. In our applications, we will need to be careful when calculating the sum $\sum R$ so that we do not count a residue of the same pole twice.

We now calculate the residues of $f(q)$ at its poles. If we let $T:=(a \tau+b) /(c \tau+d)$, then $\tau=(d T-b) /(-c T+a)$. When we substitute $T$ for $\tau$ and find a common 
denominator, we find that

$$
\begin{aligned}
A=\operatorname{Res}(\varphi(\tau), \alpha) & =\lim _{\tau \rightarrow \alpha}(c \tau+d)^{n} \varphi\left(\frac{a \tau+b}{c \tau+d}\right)(\tau-\alpha) \\
& =(c \alpha+d)^{n} \lim _{\tau \rightarrow \alpha} \varphi(T)\left(\frac{d T-b}{-c T+a}-\alpha\right) \\
& =(c \alpha+d)^{n} \lim _{T \rightarrow(a \alpha+b) /(c \alpha+d)} \varphi(T)\left(\frac{(c \alpha+d) T-(a \alpha+b)}{-c T+a}\right) \\
& =(c \alpha+d)^{n} \lim _{T \rightarrow(a \alpha+b) /(c \alpha+d)} \varphi(T)\left(\frac{T-\frac{a \alpha+b}{c \alpha+d}}{-c\left(\frac{a \alpha+b}{c \alpha+d}\right)+a}\right)(c \alpha+d) \\
(2.35) \quad & (c \alpha+d)^{n+2} \lim _{T \rightarrow(a \alpha+b) /(c \alpha+d)} \varphi(T)\left(T-\frac{a \alpha+b}{c \alpha+d}\right) .
\end{aligned}
$$

Note that the right-hand side of (2.35) is $(c \alpha+d)^{n+2}$ times the residue of $\varphi(\tau)$ at $\tau=(a \alpha+b) /(c \alpha+d)$. Hence,

$$
\operatorname{Res}\left(\varphi(\tau), \frac{a \alpha+b}{c \alpha+d}\right)=\frac{A}{(c \alpha+d)^{n+2}} .
$$

Using (2.36) and the fact that

$$
\left.\frac{d q}{d \tau}\right|_{\tau=\frac{a \alpha+b}{c \alpha+d}}=\pi i \exp \left(\pi i \frac{a \alpha+b}{c \alpha+d}\right)
$$

we find that

$$
\operatorname{Res}\left(f(q), \pm \exp \left(\pi i \frac{a \alpha+b}{c \alpha+d}\right)\right)= \pm \frac{\pi i A \exp \left(\pi i \frac{a \alpha+b}{c \alpha+d}\right)}{(c \alpha+d)^{n+2}}= \pm \frac{\pi i A \underline{q}}{(c \alpha+d)^{n+2}}
$$

where

$$
\underline{q}=\exp \left(\pi i \frac{a \alpha+b}{c \alpha+d}\right) .
$$

When we use (2.37) to evaluate the sum $-\sum R$ in (2.33), we find that (2.33) becomes

$$
\begin{aligned}
f(q) & =-\sum\left(\frac{\pi i A \underline{q}}{(c \alpha+d)^{n+2}} \frac{1}{\underline{q}-q}+\frac{-\pi i A \underline{q}}{(c \alpha+d)^{n+2}} \frac{1}{-\underline{q}-q}\right) \\
& =-2 \pi i A \sum \frac{1}{(c \alpha+d)^{n+2}} \frac{1}{1-(q / \underline{q})^{2}},
\end{aligned}
$$

where the summation runs over all pairs of coprime integers $(c, d)$ which yield distinct values for $\pm \underline{q}$ (the poles of $f(q)$ ), and $(a, b)$ is any integral solution to $a d-b c=1$. Thus the proof of the theorem is complete for $|q|<1$.

If $|q|>1$, the proof is the same except now there is not a pole of $f(q)$ inside the unit circle. Thus, the term $f(q)$ in (2.2) does not appear, and so we arrive at (2.3) instead. 


\section{The COefFicients of $1 / E_{4}$}

As we noted earlier, Hardy and Ramanujan used Theorem[2.1 to prove a formula for the coefficients in the power series expansion of the reciprocal of the Eisenstein series $E_{6}$. In this section we will prove one of the analogous results found with the publication of the lost notebook [19, pp. 102-104], 6, pp. 179-182]. These three pages are apparently taken from one of Ramanujan's letters to Hardy in 1918, but the pages are undated and bear no salutation.

Let $K=\mathbb{Q}(\sqrt{-3})$. The algebraic integral domain $\mathfrak{O}_{K}=\mathbb{Z}[\zeta]=\mathbb{Z} \oplus \zeta \mathbb{Z}$, where $\zeta=\exp (2 \pi i / 3)$, is a principal ideal domain. If $(c, d)$ is a pair of coprime integers which is a solution to the equation

$$
\lambda=c^{2}-c d+d^{2},
$$

where $\lambda$ is a certain fixed positive integer, then

$$
\pm(c, d), \quad \pm(d, c), \quad \pm(c-d, c), \quad \pm(c, c-d), \quad \pm(d, d-c), \quad \pm(c-d,-d)
$$

are solutions as well. To see this, set $\alpha=c+d \zeta$ and let $\mathfrak{A}=(c+d \zeta)$ be an ideal. Then, if $N$ denotes the norm of $\mathfrak{A}$, we see that

$$
\lambda=N(\mathfrak{A})=\mathfrak{A} \overline{\mathfrak{A}}=(c+d \zeta)(c+d \bar{\zeta})
$$

where $\bar{\zeta}=\zeta^{2}$. The unit group in $\mathfrak{D}_{K}$ is $U:=\left\{ \pm 1, \pm \zeta, \pm \zeta^{2}\right\}$. It follows that

$$
\begin{aligned}
& \mathfrak{A}=(\alpha)=(-\alpha)=(\alpha \zeta)=(-\alpha \zeta)=\left(\alpha \zeta^{2}\right)=\left(-\alpha \zeta^{2}\right), \\
& \overline{\mathfrak{A}}=(\bar{\alpha})=(-\bar{\alpha})=(\bar{\alpha} \zeta)=(-\bar{\alpha} \zeta)=\left(\bar{\alpha} \zeta^{2}\right)=\left(-\bar{\alpha} \zeta^{2}\right) .
\end{aligned}
$$

Hence, one solution generates twelve solutions. For example, if

$$
\mathfrak{A}=(\alpha \zeta)=\left(c \zeta+d \zeta^{2}\right)=(c \zeta+d(-1-\zeta))=(-d+(c-d) \zeta),
$$

then $(-d, c-d)$ is a solution if $(c, d)$ is a solution. Note that if $\lambda$ is a prime, then the twelve solutions are the only solutions, but if $\lambda$ is composite, there may be many solutions. We will say that two solutions $\left(c_{1}, d_{1}\right)$ and $\left(c_{2}, d_{2}\right)$ to equation (3.1) are distinct if they do not simultaneously belong to the same set of solutions in (3.2).

Note that each of the solutions in (3.2) has an element from two of the three sets $\{ \pm c\},\{ \pm d\}$ and $\{ \pm(c-d)\}$. Thus if two solutions simultaneously belong to (3.2), then they have an element in common at least in absolute value. It follows that $\left(c_{1}, d_{1}\right)$ and $\left(c_{2}, d_{2}\right)$ are distinct solutions to (3.1) if and only if

$$
c_{2}, d_{2} \notin\left\{ \pm c_{1}, \pm d_{1}\right\} .
$$

It is well known, see, e.g., the text by Niven, Zuckerman, and Montgomery [11. p. 176], that the integers $\lambda$ which can be represented in the form of $\lambda=c^{2}-c d+d^{2}$, with $c$ and $d$ coprime, are integers of the form

$$
\lambda=3^{a} \prod_{j=1}^{r} p_{j}^{a_{j}},
$$

where $a=0$ or $1, p_{j}$ is a prime of the form $6 m+1$, and $a_{j}$ is a nonnegative integer, $1 \leq j \leq r$.

Theorem 3.1. Recall that $Q(q)$ and $R(q)$ are defined by (1.2) and (1.3), respectively. Let

$$
\frac{1}{Q\left(q^{2}\right)}=\sum_{n=0}^{\infty} \beta_{n} q^{2 n}
$$


and

$$
G:=R\left(e^{2 \pi i \rho}\right)=1-504 \sum_{k=1}^{\infty} \frac{(-1)^{k} k^{5}}{e^{k \pi \sqrt{3}}-(-1)^{k}}=2.8815 \ldots,
$$

where $\rho:=-1 / 2+i \sqrt{3} / 2$. Then

$$
\begin{aligned}
\beta_{n}=(-1)^{n} \frac{3}{G}\left\{e^{n \pi \sqrt{3}}-\frac{e^{n \pi \sqrt{3} / 3}}{3^{3}}+\frac{2 \cos \left(\frac{2 \pi n}{7}-6 \arctan (-3 \sqrt{3})\right)}{7^{3}} e^{n \pi \sqrt{3} / 7}\right. \\
+ \\
\left.+\frac{2 \cos \left(\frac{6 \pi n}{13}-6 \arctan (-2 \sqrt{3})\right) e^{n \pi \sqrt{3} / 13}}{13^{3}}+\cdots\right\} \\
=(-1)^{n} \frac{3}{G} \sum_{(\lambda)} \frac{h_{\lambda}(n)}{\lambda^{3}} e^{n \pi \sqrt{3} / \lambda} .
\end{aligned}
$$

Here $\lambda$ runs over the integers of the form (3.4),

$$
h_{1}(n)=1, \quad h_{3}(n)=-1,
$$

and, for $\lambda \geq 7$,

$$
h_{\lambda}(n)=2 \sum_{c, d} \cos \left((a d+b c-2 a c-2 b d+\lambda) \frac{n \pi}{\lambda}-6 \arctan \left(\frac{c \sqrt{3}}{2 d-c}\right)\right),
$$

where the sum is over all pairs $(c, d)$, where $(c, d)$ is a distinct solution to $\lambda=$ $c^{2}-c d+d^{2}$ and $(a, b)$ is any solution to $a d-b c=1$. Also, distinct solutions $(c, d)$ to $\lambda=c^{2}-c d+d^{2}$ give rise to distinct terms in the sum in (3.6). Furthermore, if $n<0$, the sum on the far right side of (3.6) equals 0 .

Proof. We apply Theorem 2.1 to the function $1 / Q\left(q^{2}\right)$. Then $\varphi(\tau)=1 / Q\left(e^{2 \pi i \tau}\right)=$ $1 / E_{4}(\tau)$. Since the Eisenstein series $E_{2 j}(\tau)$ is a modular form of degree $-2 j$ [21] p. 50], $\varphi(\tau)$ satisfies the functional equation (2.1) with $n=4$, i. e.,

$$
\varphi(\tau)=\varphi\left(\frac{a \tau+b}{c \tau+d}\right)(c \tau+d)^{4}
$$

The function $\varphi(\tau)$ has only one pole in $P_{1}$, a simple pole at $\tau=-\frac{1}{2}+i \frac{\sqrt{3}}{2}=: \rho[20$, p. 198]. Thus, in (2.2), we have

$$
\alpha=\rho .
$$

Clearly $\varphi(\tau)$ is meromorphic in $\mathcal{H}$, which implies that $f(q)$ is meromorphic in the unit disc.

We now calculate $A=\operatorname{Res}(\varphi, \rho)$ by calculating the corresponding residue, $\operatorname{Res}\left(f, e^{\pi i \rho}\right)$.

Suppose that a function $F(q)$ has a simple pole at $q=q_{1}$. Expanding $F(q)$ into its Laurent series about $q=q_{1}$, we can easily see that

$$
\operatorname{Res}\left(F, q_{1}\right)=\left.\frac{1}{d(1 / F(q)) / d q}\right|_{q=q_{1}} .
$$


By (3.11), (1.2), and an identity of Ramanujan $3 q Q^{\prime}(q)=P(q) Q(q)-R(q)$ [17, [18, p. 142, Table II, no. 2],

$$
\begin{aligned}
\operatorname{Res}\left(f, e^{\pi i \rho}\right)=\left.\frac{1}{d\left(Q\left(q^{2}\right)\right) / d q}\right|_{q=e^{\pi i \rho}} & =\left.\frac{3}{2} \frac{q}{720 \sum_{k=1}^{\infty} \frac{k^{4} q^{2 k}}{\left(1-q^{2 k}\right)^{2}}}\right|_{q=e^{\pi i \rho}} \\
& =\left.\frac{3}{2} \frac{q}{P\left(q^{2}\right) Q\left(q^{2}\right)-R\left(q^{2}\right)}\right|_{q=e^{\pi i \rho}} \\
& =-\frac{3}{2} \frac{e^{\pi i \rho}}{R\left(e^{2 \pi i \rho}\right)} \\
& =-\frac{3 e^{\pi i \rho}}{2 G},
\end{aligned}
$$

where $G$ is given by (3.5).

If we apply (2.37) with $\alpha=\rho$ and $(a, b, c, d)=(1,0,0,1)$, then we deduce that

$$
\pi i A e^{\pi i \rho}=-\frac{3 e^{\pi i \rho}}{2 G}
$$

or

$$
A=-\frac{3}{2 \pi i G} .
$$

By (2.2), (3.9), (3.10), and (3.12), we find that

$$
f(q)=\frac{3}{G} \sum_{(c, d)} \frac{1}{(c \rho+d)^{6}} \frac{1}{1-(q / \underline{q})^{2}},
$$

where

$$
\underline{q}=\exp \left(\pi i\left(\frac{a \rho+b}{c \rho+d}\right)\right),
$$

and where the summation runs over all pairs of coprime integers $(c, d)$ which produce distinct values for the set $\{\underline{q},-\underline{q}\}$, and $(a, b)$ is any integral solution to $a d-b c=1$.

By (2.34), each pair $(c, \bar{d})$ leads to exactly two distinct poles of $f$ in the unit circle, $\underline{q}$ and $-\underline{q}$, but it is possible that different pairs may lead to the same poles, so we need to be careful that we do not count the same pole twice in the summation.

Thus, two tasks remain: find the values of $(c, d)$ over which the summation runs, and compare the coefficients of $q^{n}$ on both sides of (3.13).

First, if $\lambda=c^{2}-c d+d^{2}$,

$$
\begin{aligned}
\frac{a \rho+b}{c \rho+d}=\frac{(a \rho+b)\left(c \rho^{2}+d\right)}{\lambda} & =\frac{a c+b d+(a d-b c) \rho+b c\left(\rho+\rho^{2}\right)}{\lambda} \\
& =\frac{a c+b d-b c-\frac{1}{2}+\frac{\sqrt{3}}{2} i}{\lambda} .
\end{aligned}
$$

So,

$$
\underline{q}=\exp \left(\frac{-\pi \sqrt{3}}{2 \lambda}\right) \exp \left(\frac{\pi i}{\lambda}\left(a c+b d-\frac{1}{2} a d-\frac{1}{2} b c\right)\right) .
$$


If two pairs $\left(c_{3}, d_{3}\right)$ and $\left(c_{4}, d_{4}\right)$ produce distinct values of $\lambda$, i.e., $c_{3}^{2}-c_{3} d_{3}+d_{3}^{2}=$ $\lambda_{3} \neq \lambda_{4}=c_{4}^{2}-c_{4} d_{4}+d_{4}^{2}$, then those pairs lead to distinct values for the set $\{ \pm \underline{q}\}$, say $\left\{ \pm \underline{q}_{3}\right\}$ and $\left\{ \pm \underline{q}_{4}\right\}$, i.e.,

$$
\left\{ \pm \underline{q}_{3}\right\} \wedge\left\{ \pm \underline{q}_{4}\right\}=\emptyset .
$$

We now consider the case when different values of $(c, d)$ produce the same values of $\lambda$. As we saw in (3.2), each solution $(c, d)$ to $\lambda=c^{2}-c d+d^{2}$ generates a total of twelve solutions. If $\lambda=1$ or $\lambda=3$, then only six of these twelve are different solutions. If $\lambda \geq 7$, then the twelve solutions are all different.

Suppose that the solution $(c, d)$ leads to $\left\{ \pm_{q_{5}}\right\}$, say. Then $(-c,-d)$ also leads to $\left\{ \pm \underline{q}_{5}\right\}$, while $(d, c)$ and $(c, c-d)$ both lead to $\left\{ \pm \underline{q}_{5}\right\}$. Since these three basic transformations lead to either $\left\{ \pm q_{5}\right\}$ or $\left\{ \pm \bar{q}_{5}\right\}$, it follows that $(c, d)$ and the eleven corresponding solutions of (3.2) yield a set of only four different poles, namely,

$$
\left\{ \pm \underline{q}_{5}, \pm \underline{q}_{5}\right\} .
$$

This would be a set of only two poles if $\underline{q}_{5}$ were real or purely imaginary. We will prove in Lemmas 3.2, 3.3 and 3.4 at the end of this section that $q$ can never be real, and that it is purely imaginary only when $\lambda=1$ or $\lambda=3$. Thus, (3.17) is valid only for $\lambda \geq 7$. If $\lambda=1$ or $\lambda=3$, then the solutions of (3.2) produce a set of only two poles (for each value of $\lambda$ ),

$$
\left\{ \pm \underline{q}_{5}\right\} .
$$

Lastly, suppose that $\left(c_{1}, d_{1}\right)$ and $\left(c_{2}, d_{2}\right)$ are distinct solutions to equation (3.1). In Lemma 3.5 at the end of the section, we will prove that each distinct solution (together with its eleven corresponding solutions in (3.2) yields four distinct poles, i.e.,

$$
\left\{ \pm \underline{q}_{1}, \pm \underline{\bar{q}}_{1}\right\} \wedge\left\{ \pm \underline{q}_{2}, \pm \underline{\bar{q}}_{2}\right\}=\emptyset,
$$

where the bracketed sets correspond to $\left(c_{1}, d_{1}\right)$ and $\left(c_{2}, d_{2}\right)$, respectively.

We can now express the right-hand side of (3.13) not as a sum over pairs $(c, d)$, but as a sum over $\lambda$ and over distinct pairs $(c, d)$.

From (3.13) and (3.16)-(3.19),

$$
\begin{aligned}
f(q)= & \frac{3}{G}\left\{\sum_{\substack{(\lambda) \\
\lambda \leq 3}} \frac{1}{(c \rho+d)^{6}} \frac{1}{1-(q / \underline{q})^{2}}\right. \\
& \left.+\sum_{\substack{(\lambda) \\
\lambda>3}}\left(\frac{1}{(c \rho+d)^{6}} \frac{1}{1-(q / \underline{q})^{2}}+\frac{1}{(d \rho+c)^{6}} \frac{1}{1-(q / \bar{q})^{2}}\right)\right\},
\end{aligned}
$$

where $\lambda$ runs over all integers of the form (3.4), and where, for each fixed $\lambda$, the sum is also over all distinct pairs $(c, d)$.

For $\lambda=1$, with $(a, b, c, d)=(1,0,0,1)$, by (3.15),

$$
\underline{q}=\exp (-\pi \sqrt{3} / 2) \exp (-\pi i / 2)=-i e^{-\pi \sqrt{3} / 2} .
$$


Thus,

$$
\frac{1}{(c \rho+d)^{6}} \frac{1}{1-(q / \underline{q})^{2}}=\frac{1}{1+e^{\pi \sqrt{3}} q^{2}} .
$$

Similarly, for $\lambda=3$, with $(a, b, c, d)=(1,1,1,2)$, by (3.15),

$$
\underline{q}=\exp \left(\frac{-\pi \sqrt{3}}{6}\right) \exp \left(\frac{\pi i}{3}\left(\frac{3}{2}\right)\right)=i \exp \left(\frac{-\pi \sqrt{3}}{6}\right) .
$$

Thus,

$$
\frac{1}{(c \rho+d)^{6}} \frac{1}{1-(q / \underline{q})^{2}}=-\frac{1}{27} \frac{1}{1+e^{\pi \sqrt{3} / 3} q^{2}} .
$$

By (3.21) and (3.22), equality (3.20) becomes

$$
\begin{aligned}
f(q)= & \frac{3}{G}\left\{\frac{1}{1+e^{\pi \sqrt{3}} q^{2}}-\frac{1}{27} \frac{1}{1+e^{\pi \sqrt{3} / 3} q^{2}}\right. \\
& \left.+\sum_{\substack{(\lambda) \\
\lambda>3}}\left(\frac{1}{(c \rho+d)^{6}} \frac{1}{1-(q / \underline{q})^{2}}+\frac{1}{(d \rho+c)^{6}} \frac{1}{1-(q / \underline{\bar{q}})^{2}}\right)\right\} \\
= & \frac{3}{G}\left\{\sum_{n=0}^{\infty}(-1)^{n} e^{n \pi \sqrt{3}} q^{2 n}-\frac{1}{27} \sum_{n=0}^{\infty}(-1)^{n} e^{n \pi \sqrt{3} / 3} q^{2 n}\right. \\
& \left.+\sum_{(\lambda)}\left(\frac{1}{(c \rho+d)^{6}} \sum_{n=0}^{\infty} \underline{q}^{-2 n} q^{2 n}+\frac{1}{(d \rho+c)^{6}} \sum_{n=0}^{\infty} \underline{\underline{q}}^{-2 n} q^{2 n}\right)\right\} \\
= & \sum_{n=0}^{\infty} \beta_{n} q^{2 n},
\end{aligned}
$$

where $|q|<e^{-\pi \sqrt{3} / 2}$ and

$$
\beta_{n}=(-1)^{n} \frac{3}{G}\left(e^{n \pi \sqrt{3}}-\frac{e^{n \pi \sqrt{3} / 3}}{3^{3}}\right)+\frac{3}{G} \sum_{\substack{(\lambda) \\ \lambda>3}}\left(\frac{1}{(c \rho+d)^{6}} \underline{q}^{-2 n}+\frac{1}{(d \rho+c)^{6}} \underline{\bar{q}}^{-2 n}\right) .
$$

We now show that

$$
\frac{1}{(c \rho+d)^{6}}=\left(\overline{\frac{1}{(d \rho+c)^{6}}}\right),
$$

and then we will use this to express the sum in (3.25) more explicitly. By an elementary calculation, we find that

$$
\frac{1}{(c \rho+d)^{6}}=\frac{1}{\lambda^{3}} \exp \left(-6 i \arctan \left(\frac{c \sqrt{3}}{2 d-c}\right)\right),
$$

and similarly,

$$
\frac{1}{(d \rho+c)^{6}}=\frac{1}{\lambda^{3}} \exp \left(-6 i \arctan \left(\frac{d \sqrt{3}}{2 c-d}\right)\right) \text {. }
$$


Note, however, that

$$
\tan \left(\arctan \left(\frac{c \sqrt{3}}{2 d-c}\right)+\arctan \left(\frac{d \sqrt{3}}{2 c-d}\right)\right)=\frac{\frac{c \sqrt{3}}{2 d-c}+\frac{d \sqrt{3}}{2 c-d}}{1-\left(\frac{c \sqrt{3}}{2 d-c}\right)\left(\frac{d \sqrt{3}}{2 c-d}\right)}=-\sqrt{3}
$$

Hence,

$$
\arctan \left(\frac{c \sqrt{3}}{2 d-c}\right)+\arctan \left(\frac{d \sqrt{3}}{2 c-d}\right)=m \pi-\frac{\pi}{3}
$$

where $m$ is some integer. Thus from (3.27), (3.28), and (3.29),

$$
\frac{1}{(c \rho+d)^{6}} \frac{1}{(d \rho+c)^{6}}=\frac{1}{\lambda^{6}} \exp \left(-6 i\left(m \pi-\frac{\pi}{3}\right)\right)=\frac{1}{\lambda^{6}},
$$

which is, of course, real. Since $\frac{1}{(c \rho+d)^{6}} \frac{1}{(d \rho+c)^{6}}$ is real, (3.26) follows. From (3.15),

$$
\underline{q}^{-2 n}=(-1)^{n} \exp \left(\frac{n \pi \sqrt{3}}{\lambda}\right) \exp \left(\frac{n \pi i}{\lambda}(a d+b c-2 a c-2 b d+\lambda)\right) .
$$

Thus, by (3.30), 3.27), and (3.31), each summand in the sum of 3.25) is

$$
\begin{aligned}
2 & \operatorname{Re}\left(\frac{\underline{q}^{-2 n}}{(c \rho+d)^{6}}\right) \\
& =2(-1)^{n} e^{n \pi \sqrt{3} / \lambda} \\
& \times \operatorname{Re}\left(\frac{\exp \left(\frac{n \pi i}{\lambda}(a d+b c-2 a c-2 b d+\lambda)\right) \exp \left(-6 i \arctan \left(\frac{c \sqrt{3}}{2 d-c}\right)\right)}{\lambda^{3}}\right) \\
& =\frac{(-1)^{n}}{\lambda^{3}} 2 \cos \left((a d+b c-2 a c-2 b d+\lambda) \frac{n \pi}{\lambda}-6 \arctan \left(\frac{c \sqrt{3}}{2 d-c}\right)\right) e^{n \pi \sqrt{3} / \lambda} .
\end{aligned}
$$


From (3.25) and (3.32), the coefficient of $q^{2 n}$ in the power series expansion of $f(q)$ is

$$
\begin{aligned}
& \beta_{n}=(-1)^{n} \frac{3}{G}\left\{e^{n \pi \sqrt{3}}-\frac{e^{n \pi \sqrt{3} / 3}}{3^{3}}\right. \\
& \left.+\sum_{(\lambda)} \frac{2 \cos \left((a d+b c-2 a c-2 b d+\lambda) \frac{n \pi}{\lambda}-6 \arctan \left(\frac{c \sqrt{3}}{2 d-c}\right)\right)}{\lambda^{3}} e^{n \pi \sqrt{3} / \lambda}\right\} \\
& (3.33) \quad=(-1)^{n} \frac{3}{G} \sum_{(\lambda)} \frac{h_{\lambda}(n)}{\lambda^{3}} e^{n \pi \sqrt{3} / \lambda},
\end{aligned}
$$

where $h_{\lambda}(n)$ is defined in (3.7) and (3.8). This proves (3.6).

To obtain the displayed terms in the expansion $(\underline{3.6})$, we choose $(a, b, c, d)=$ $(1,0,3,1)$ for $\lambda=7$ and $(a, b, c, d)=(1,0,4,1)$ for $\lambda=13$.

Lastly, we consider the case for $n<0$. Up until (3.23), we did not use the fact that $|q|<1$, except that, if $|q|>1$, by Theorem 2.1, the left side of (3.23) would equal 0 . Instead of expanding the summands on the left side of (3.23) in powers of $q$, we expand the summands in powers of $q^{-1}$ when $|q|>1$. We thus find that, for $|q|>e^{\pi \sqrt{3} / 2}$

$$
\begin{aligned}
0= & \frac{3}{G}\left\{\sum_{n=1}^{\infty}(-1)^{n} e^{-n \pi \sqrt{3}} q^{-2 n}-\frac{1}{27} \sum_{n=1}^{\infty}(-1)^{n} e^{-n \pi \sqrt{3} / 3} q^{-2 n}\right. \\
& \left.+\sum_{\substack{(\lambda) \\
\lambda>3}}\left(\frac{1}{(c \rho+d)^{6}} \sum_{n=1}^{\infty} \underline{q}^{2 n} q^{-2 n}+\frac{1}{(d \rho+c)^{6}} \sum_{n=1}^{\infty} \underline{q}^{2 n} q^{-2 n}\right)\right\} \\
= & \sum_{n=1}^{\infty} \beta_{-n} q^{-2 n} .
\end{aligned}
$$

This then completes the proof of the theorem for $n<0$.

Thus the proof of Theorem 3.1 is complete apart from several technical lemmas.

Lemma 3.2. Given a coprime pair of integers $(c, d)$, we can always choose integers $a$ and $b$ such that $a d-b c=1$ and

$$
\left|a c+b d-\frac{1}{2}(a d+b c)\right| \leq \frac{1}{2}\left(c^{2}-c d+d^{2}\right) .
$$

Proof. Let $\left(a_{1}, b_{1}\right)$ be a solution to $a d-b c=1$. Then the complete set of solutions is $\left\{\left(a_{1}+m c, b_{1}+m d\right): m \in \mathbf{Z}\right\}$. Substituting this expression into the left side of (3.34), we see that

$$
\begin{aligned}
& \left|\left(a_{1}+m c\right) c+\left(b_{1}+m d\right) d-\frac{1}{2}\left(a_{1}+m c\right) d-\frac{1}{2}\left(b_{1}+m d\right) c\right| \\
& =\left|a_{1} c+b_{1} d-\frac{1}{2} a_{1} d-\frac{1}{2} b_{1} c+m\left(c^{2}-c d+d^{2}\right)\right| .
\end{aligned}
$$


For some unique integer $m_{1}$, we have

$$
a_{1} c+b_{1} d-\frac{1}{2} a_{1} d-\frac{1}{2} b_{1} c+m_{1}\left(c^{2}-c d+d^{2}\right) \leq 0
$$

and

$$
a_{1} c+b_{1} d-\frac{1}{2} a_{1} d-\frac{1}{2} b_{1} c+\left(m_{1}+1\right)\left(c^{2}-c d+d^{2}\right) \geq 0,
$$

since $c^{2}-c d+d^{2}>0$. Thus one of the two pairs,

$$
a=a_{1}+m_{1} c, \quad b=b_{1}+m_{1} d \quad \text { or } \quad a=a_{1}+\left(m_{1}+1\right) c, \quad b=b_{1}+\left(m_{1}+1\right) d
$$

will be our desired solution.

Lemma 3.3. If $a d-b c=1$, where $a, b, c, d \in \mathbf{Z}$, then the quantity

$$
\underline{q}=\exp \left(\frac{-\pi \sqrt{3}}{2\left(c^{2}-c d+d^{2}\right)}\right) \exp \left(\pi i\left(\frac{a c+b d-\frac{1}{2}(a d+b c)}{c^{2}-c d+d^{2}}\right)\right)
$$

cannot be real.

Proof. By (2.34), if $c$ and $d$ are fixed and $(a, b)$ is a solution to $a d-b c=1$ which leads to $\underline{q}$, then other solutions to $a d-b c=1$ will lead to either $\underline{q}$ or $-\underline{q}$. Therefore if $q$ is real for some solution $(a, b)$, then it is real for all solutions.

Suppose that a certain pair $(c, d)$ leads to a value of $q$ that is real. We can assume without loss of generality that $(a, b)$ satisfies (3.34). Since $\underline{q}$ is real, we have, by (3.35),

$$
a c+b d-\frac{1}{2}(a d+b c) \equiv 0 \quad\left(\bmod \left(c^{2}-c d+d^{2}\right)\right),
$$

and so, by (3.34),

$$
a c+b d-\frac{1}{2}(a d+b c)=0 .
$$

Adding the equations $0=\left(a c+b d-\frac{1}{2}(a d+b c)\right)^{2}$ and $1=(a d-b c)^{2}$ gives

$$
\begin{aligned}
1= & a^{2} c^{2}+a^{2} d^{2}+b^{2} c^{2}+b^{2} d^{2}-a^{2} c d-a b c^{2}-a b d^{2}-b^{2} c d \\
& +\frac{1}{4}\left(a^{2} d^{2}+2 a b c d+b^{2} c^{2}\right) \\
= & \left(c^{2}-c d+d^{2}\right)\left(a^{2}-a b+b^{2}\right)+\frac{1}{4}(a d-b c)^{2} \\
= & \left(c^{2}-c d+d^{2}\right)\left(a^{2}-a b+b^{2}\right)+\frac{1}{4}
\end{aligned}
$$

Hence,

$$
\frac{3}{4}=\left(c^{2}-c d+d^{2}\right)\left(a^{2}-a b+b^{2}\right),
$$

which is impossible since the variables are integers. Thus $\underline{q}$ cannot be real.

Lemma 3.4. Under the conditions of Lemma 3.3, the quantity $\underline{q}$ is purely imaginary only when $\lambda=1$ or $\lambda=3$, where $\lambda=c^{2}-c d+d^{2}$.

Proof. Suppose that a certain pair $(c, d)$ leads to a value of $\underline{q}$ that is purely imaginary. We can assume without loss of generality that $(a, b)$ satisfies (3.34). Since $\underline{q}$ is purely imaginary, we have, by (3.35),

$$
2\left|a c+b d-\frac{1}{2}(a d+b c)\right|=c^{2}-c d+d^{2} .
$$


Thus, by (3.36) and the equality $a d-b c=1$,

$$
\begin{aligned}
& \left(\left|a c+b d-\frac{1}{2}(a d+b c)\right|-\left(a^{2}-a b+b^{2}\right)\right)^{2} \\
= & \left(a c+b d-\frac{1}{2}(a d+b c)\right)^{2}-2\left|a c+b d-\frac{1}{2}(a d+b c)\right|\left(a^{2}-a b+b^{2}\right) \\
& +\left(a^{2}-a b+b^{2}\right)^{2} \\
= & \left(a c+b d-\frac{1}{2}(a d+b c)\right)^{2}-\left(c^{2}-c d+d^{2}\right)\left(a^{2}-a b+b^{2}\right)+\left(a^{2}-a b+b^{2}\right)^{2} \\
= & \left(a^{2}-a b+b^{2}\right)^{2}-\frac{3}{4}(a d-b c)^{2} \\
= & \left(a^{2}-a b+b^{2}\right)^{2}-\frac{3}{4} \\
= & \Lambda^{2}-\frac{3}{4},
\end{aligned}
$$

where

Since $\Lambda \in \mathbf{Z}$,

$$
\left|a c+b d-\frac{1}{2}(a d+b c)\right|=\frac{1}{2} W,
$$

where $W$ is odd and positive. Therefore, by (3.37) and (3.38),

$$
\left(\frac{1}{2} W-\Lambda\right)^{2}=\Lambda^{2}-\frac{3}{4}
$$

or

$$
W\left(\frac{1}{4} W-\Lambda\right)=-\frac{3}{4} .
$$

Hence, the quantity $\frac{1}{4} W-\Lambda$ is negative. Clearly, its absolute value is at least $\frac{1}{4}$. Since

$$
\frac{3}{4}=\left|W\left(\frac{1}{4} W-\Lambda\right)\right| \geq\left|\frac{1}{4} W\right|,
$$

we deduce that $W=1$ or $W=3$. But by (3.38) and (3.36),

$$
W=2\left|a c+b d-\frac{1}{2}(a d+b c)\right|=c^{2}-c d+d^{2}=\lambda .
$$

We conclude that if $\underline{q}$ is purely imaginary, then $\lambda$ can be only 1 or 3 . When $\lambda=1$ and $(c, d)=(1,0)$, say, we have, from 3.35),

$$
\underline{q}= \pm e^{-\pi \sqrt{3} / 2} e^{\pi i / 2}= \pm i e^{-\pi \sqrt{3} / 2}
$$

When $\lambda=3$ and $(c, d)=(2,1)$, say, we have

$$
\underline{q}= \pm e^{-\pi /(2 \sqrt{3})} e^{-\pi i / 2}=\mp i e^{-\pi /(2 \sqrt{3})} .
$$

So if $\lambda=1$ or $\lambda=3$, the quantity $\underline{q}$ is indeed purely imaginary.

In Lemma 3.5, we establish 3.19 by proving the contrapositive statement, namely, that if two different solutions $(c, d)$ to the equation $\lambda=c^{2}-c d+d^{2}$ lead to the same set of poles, then the solutions are not distinct.

Lemma 3.5. If $\left(c_{6}, d_{6}\right)$ and $\left(c_{7}, d_{7}\right)$ are two pairs of coprime integers such that

$$
c_{6}^{2}-c_{6} d_{6}+d_{6}^{2}=c_{7}^{2}-c_{7} d_{7}+d_{7}^{2}=\lambda,
$$

and if

$$
\left\{ \pm \underline{q}_{6}, \pm \underline{\bar{q}}_{6}\right\}=\left\{ \pm \underline{q}_{7}, \pm \underline{\bar{q}}_{7}\right\}
$$

where $\underline{q}$ is defined in (3.15), then the two solutions of (3.39) are not distinct. In other words,

$$
\left\{c_{7}, d_{7}\right\} \wedge\left\{ \pm c_{6}, \pm d_{6}\right\} \neq \emptyset \text {. }
$$


Proof. From (2.34), we see that for fixed $\left(c_{j}, d_{j}\right)$, the set $\left\{ \pm \underline{q}_{j}, \pm \underline{\bar{q}}_{j}\right\}$ is not affected by our choice of $\left(a_{j}, b_{j}\right)$. Thus we can assume without loss of generality that $\left(a_{j}, b_{j}\right)$ satisfies (3.34) for $j=6$ and 7 . In other words, if we define $e_{j}$ by

$$
e_{j}=a_{j} c_{j}+b_{j} d_{j}-\frac{1}{2} a_{j} d_{j}-\frac{1}{2} b_{j} c_{j}
$$

then we can assume that

$$
\left|e_{j}\right| \leq \frac{1}{2} \lambda, \quad j=6,7 .
$$

We will show that (3.40) implies $e_{6}= \pm e_{7}$ by considering four different cases.

If $\underline{q}_{6}=\underline{q}_{7}$, then by (3.15) and (3.42), $\pi i e_{6} / \lambda=\pi i e_{7} / \lambda+2 \pi i m$, where $m$ is some integer. In other words,

$$
e_{6} \equiv e_{7} \quad(\bmod 2 \lambda) \text {. }
$$

If, however, $\underline{q}_{6}=\underline{\bar{q}}_{7}$, then, by (3.15) and (3.42), $\pi i e_{6} / \lambda=-\pi i e_{7} / \lambda+2 \pi i m_{1}$, where $m_{1}$ is some integer. Thus,

$$
e_{6} \equiv-e_{7} \quad(\bmod 2 \lambda) .
$$

If $\underline{q}_{6}=-\underline{q}_{7}$, then, by (3.15) and (3.42), $\pi i e_{6} / \lambda=\pi i e_{7} / \lambda+\pi i+2 \pi i m_{2}$, where $m_{2}$ is some integer, or, in other words,

$$
e_{6} \equiv e_{7}+\lambda \quad(\bmod 2 \lambda) \text {. }
$$

Similarly, if $\underline{q}_{6}=-\underline{\underline{q}}_{7}$, then $\pi i e_{6} / \lambda=-\pi i e_{7} / \lambda+\pi i+2 \pi i m_{3}$, where $m_{3}$ is some integer, which implies that

$$
e_{6} \equiv-e_{7}+\lambda \quad(\bmod 2 \lambda) .
$$

From (3.44)-3.47), we see that the set equality $\left\{ \pm \underline{q}_{6}, \pm \underline{\bar{q}}_{6}\right\}=\left\{ \pm \underline{q}_{7}, \pm \underline{\bar{q}}_{7}\right\}$ implies that $e_{6} \equiv \pm e_{7}(\bmod \lambda)$, which implies that, by (3.43),

$$
e_{6}= \pm e_{7}
$$

Observe that, by (2.4) and (3.42),

$$
e_{j}^{2}+1=e_{j}^{2}+\left(a_{j} d_{j}-b_{j} c_{j}\right)^{2}=\left(c_{j}^{2}-c_{j} d_{j}+d_{j}^{2}\right)\left(a_{j}^{2}-a_{j} b_{j}+b_{j}^{2}\right)+\frac{1}{4} .
$$

Therefore since $e_{6}^{2}=e_{7}^{2}$ and $c_{6}^{2}-c_{6} d_{6}+d_{6}^{2}=c_{7}^{2}-c_{7} d_{7}+d_{7}^{2}$, we deduce that

$$
a_{6}^{2}-a_{6} b_{6}+b_{6}^{2}=a_{7}^{2}-a_{7} b_{7}+b_{7}^{2} .
$$

Later we will use this observation.

We now prove (3.41) by using matrices. We consider two cases.

Case 1. Assume that $e_{6}=e_{7}$. If we let, for $j=1,2$,

$$
M_{j}:=\left[\begin{array}{cc}
c_{j}-\frac{1}{2} d_{j} & \frac{\sqrt{3}}{2} d_{j} \\
a_{j}-\frac{1}{2} b_{j} & \frac{\sqrt{3}}{2} b_{j}
\end{array}\right]
$$

then

$$
M_{j} M_{j}^{T}=\left[\begin{array}{cc}
c_{j}^{2}-c_{j} d_{j}+d_{j}^{2} & a_{j} c_{j}+b_{j} d_{j}-\frac{1}{2} a_{j} d_{j}-\frac{1}{2} b_{j} c_{j} \\
a_{j} c_{j}+b_{j} d_{j}-\frac{1}{2} a_{j} d_{j}-\frac{1}{2} b_{j} c_{j} & a_{j}^{2}-a_{j} b_{j}+b_{j}^{2}
\end{array}\right] .
$$

Observe that

$$
M_{6} M_{6}^{T}=M_{7} M_{7}^{T}
$$


by (3.39), (3.42), (3.49), and the assumption that $e_{6}=e_{7}$. After multiplying both sides of (3.52) by $M_{7}^{-1}$ on the left side and then by $\left(M_{6}^{T}\right)^{-1}$ on the right side, we obtain

$$
U:=M_{7}^{-1} M_{6}=M_{7}^{T}\left(M_{6}^{T}\right)^{-1}=\left(M_{6}^{-1} M_{7}\right)^{T}=\left(U^{-1}\right)^{T} .
$$

We want to determine the entries of $U$, because these may give us information about the entries of the matrices $M_{6}$ and $M_{7}$. We start by calculating the values of the determinants $\left|M_{j}\right|$ and $|U|$. From the definition of $M_{j}$ in (3.50), a straightforward calculation gives

$$
\left|M_{j}\right|=-\frac{\sqrt{3}}{2},
$$

by (2.4). Thus, by (3.53) and (3.54),

$$
|U|=\left(-\frac{2}{\sqrt{3}}\right)\left(-\frac{\sqrt{3}}{2}\right)=1 .
$$

If

$$
U=\left[\begin{array}{ll}
w & x \\
y & z
\end{array}\right]
$$

then, by (3.55) and so (3.53), we find that

$$
\left[\begin{array}{ll}
w & x \\
y & z
\end{array}\right]=\left[\begin{array}{cc}
z & -y \\
-x & w
\end{array}\right]
$$

Thus, $U$ is of the form

$$
U=\left[\begin{array}{cc}
w & x \\
-x & w
\end{array}\right]
$$

By (3.53), 3.54), and a straightforward calculation,

$U=\left[\begin{array}{cc}-b_{7}\left(c_{6}-\frac{1}{2} d_{6}\right)+d_{7}\left(a_{6}-\frac{1}{2} b_{6}\right) & -\frac{\sqrt{3}}{2} b_{7} d_{6}+\frac{\sqrt{3}}{2} b_{6} d_{7} \\ \frac{2}{\sqrt{3}}\left(a_{7}-\frac{1}{2} b_{7}\right)\left(c_{6}-\frac{1}{2} d_{6}\right)-\frac{2}{\sqrt{3}}\left(a_{6}-\frac{1}{2} b_{6}\right)\left(c_{7}-\frac{1}{2} d_{7}\right) & d_{6}\left(a_{7}-\frac{1}{2} b_{7}\right)-b_{6}\left(c_{7}-\frac{1}{2} d_{7}\right)\end{array}\right]$.

Thus, we see that $U$ has the form

$$
U=\left[\begin{array}{cc}
\frac{1}{2} P & \frac{\sqrt{3}}{2} Q \\
\frac{1}{2 \sqrt{3}} R & \frac{1}{2} S
\end{array}\right],
$$

where $P, Q, R, S \in \mathbf{Z}$. By (3.56) and (3.58), we conclude that $U$ is of the form

$$
U=\left[\begin{array}{cc}
\frac{1}{2} P & \frac{\sqrt{3}}{2} Q \\
-\frac{\sqrt{3}}{2} Q & \frac{1}{2} P
\end{array}\right],
$$

where $P, Q \in \mathbf{Z}$.

By (3.55) and (3.59), we deduce that

$$
\frac{1}{4} P^{2}+\frac{3}{4} Q^{2}=1
$$

so either $P, Q \in\{ \pm 1\}$ or $P= \pm 2$ and $Q=0$. 
If $P= \pm 2$ and $Q=0$, then

$$
U= \pm\left[\begin{array}{ll}
1 & 0 \\
0 & 1
\end{array}\right]
$$

But, by (3.53), we deduce that

$$
M_{6}= \pm M_{7},
$$

which implies that $d_{6}= \pm d_{7}$. Thus, the statement we wanted to prove, (3.41), holds.

If, however, $P, Q \in\{ \pm 1\}$, then $U$ is of the form

$$
U=\left[\begin{array}{cc} 
\pm \frac{1}{2} & \frac{\sqrt{3}}{2} \\
-\frac{\sqrt{3}}{2} & \pm \frac{1}{2}
\end{array}\right] \quad \text { or } \quad U=\left[\begin{array}{cc} 
\pm \frac{1}{2} & -\frac{\sqrt{3}}{2} \\
\frac{\sqrt{3}}{2} & \pm \frac{1}{2}
\end{array}\right] .
$$

We consider two subcases.

Case 1A. Assume the first case in (3.62). By the definition of $U$ in (3.53),

$$
\left[\begin{array}{cc}
c_{6}-\frac{1}{2} d_{6} & \frac{\sqrt{3}}{2} d_{6} \\
a_{6}-\frac{1}{2} b_{6} & \frac{\sqrt{3}}{2} b_{6}
\end{array}\right]=\left[\begin{array}{ll}
c_{7}-\frac{1}{2} d_{7} & \frac{\sqrt{3}}{2} d_{7} \\
a_{7}-\frac{1}{2} b_{7} & \frac{\sqrt{3}}{2} b_{7}
\end{array}\right]\left[\begin{array}{cc} 
\pm \frac{1}{2} & \frac{\sqrt{3}}{2} \\
-\frac{\sqrt{3}}{2} & \pm \frac{1}{2}
\end{array}\right] .
$$

The entry in the first row and second column of the matrix on the left-hand side of the equation is

$$
\frac{\sqrt{3}}{2} d_{6}=\frac{\sqrt{3}}{2}\left(c_{7}-\frac{1}{2} d_{7}\right) \pm \frac{\sqrt{3}}{4} d_{7}=\frac{\sqrt{3}}{2} c_{7}+\left(-\frac{\sqrt{3}}{4} \pm \frac{\sqrt{3}}{4}\right) d_{7} .
$$

If we choose the plus sign in the first matrix of (3.62) (and, hence, in (3.64)), then we conclude that $d_{6}=c_{7}$, from which (3.41) follows. If, however, we choose the minus sign in (3.62), then we conclude that $d_{6}=c_{7}-d_{7}$. We will show that this implies that $c_{6}=c_{7}$ or $c_{6}=-d_{7}$, from which (3.41) follows.

The pairs $\left(c_{6}, d_{6}\right)$ and $\left(c_{7}, d_{7}\right)$ are solutions to the equation $\lambda=c^{2}-c d+d^{2}$. Note that, if $\lambda$ and $d_{6}$ are fixed, then there are at most two solutions $c_{6}$ to the equation. If we set $d_{6}=c_{7}-d_{7}$, then it follows from (3.2) that two solutions for $c_{6}$ (indeed, the only two possible solutions for $c_{6}$ ) are $c_{6}=c_{7}$ or $c_{6}=-d_{7}$. It follows that (3.41) holds, and the proof for Case 1A is complete.

Case 1B. The proof for Case 1B is very similar to that for Case 1A. Assume that the second case in (3.62) holds. Note that

$$
U=\left[\begin{array}{cc} 
\pm \frac{1}{2} & -\frac{\sqrt{3}}{2} \\
\frac{\sqrt{3}}{2} & \pm \frac{1}{2}
\end{array}\right]=-\left[\begin{array}{cc}
\mp \frac{1}{2} & \frac{\sqrt{3}}{2} \\
-\frac{\sqrt{3}}{2} & \mp \frac{1}{2}
\end{array}\right],
$$

which is the matrix in Case $1 \mathrm{~A}$ multiplied by the scalar -1 . Thus, by (3.63) or (3.64),

$$
\frac{\sqrt{3}}{2} d_{6}=-\left(\frac{\sqrt{3}}{2} c_{7}+\left(-\frac{\sqrt{3}}{4} \pm \frac{\sqrt{3}}{4}\right) d_{7}\right),
$$

which implies that $d_{6}=-c_{7}$ or $d_{6}=-c_{7}+d_{7}$. But note that $d_{6}=d_{7}-c_{7}$ would imply that $c_{6}=-c_{7}$ or $c_{6}=d_{7}$ by (3.2). Hence, (3.41) follows, and the proof for Case $1 \mathrm{~B}$ is complete. 
Case 2. The proof of Case 2 is very similar to that of Case 1. Assume that

$$
e_{6}=-e_{7}
$$

If we let

$$
\tilde{M}_{7}=\left[\begin{array}{cc}
c_{7}-\frac{1}{2} d_{7} & -\frac{\sqrt{3}}{2} d_{7} \\
-\left(a_{7}-\frac{1}{2} b_{7}\right) & \frac{\sqrt{3}}{2} b_{7}
\end{array}\right],
$$

then a brief calculation gives

$$
\tilde{M}_{7} \tilde{M}_{7}^{T}=\left[\begin{array}{cc}
-c_{7}^{2}-c_{7} d_{7}+d_{7}^{2} & -\left(a_{7} c_{7}+b_{7} d_{7}-\frac{1}{2} a_{7} d_{7}-\frac{1}{2} b_{7} c_{7}\right) \\
-\left(a_{7} c_{7}+b_{7} d_{7}-\frac{1}{2} a_{7} d_{7}-\frac{1}{2} b_{7} c_{7}\right) & a_{7}^{2}-a_{7} b_{7}+b_{7}^{2}
\end{array}\right] .
$$

Note that the definition of $\tilde{M}_{7}$ is the same as that of $M_{7}$ in (3.50), except that the entries along one diagonal are multiplied by -1 . A straightforward calculation gives

$$
\left|\tilde{M}_{7}\right|=-\frac{\sqrt{3}}{2} .
$$

By (3.51), (3.39), (3.49), and (3.66), $M_{6} M_{6}^{T}=\tilde{M}_{7} \tilde{M}_{7}^{T}$ or

$$
\tilde{U}:=\tilde{M}_{7}^{-1} M_{6}=\left(M_{6}^{-1} \tilde{M}_{7}\right)^{T} \text {. }
$$

Then, from the definitions of $M_{6}$ in (3.50) and $\tilde{M}_{7}$ in (3.67) and the value of $\left|\tilde{M}_{7}\right|$ from (3.68) we find that, after multiplying the requisite matrices,

$$
\tilde{U}=\left[\begin{array}{cc}
-b_{7}\left(c_{6}-\frac{1}{2} d_{6}\right)-d_{7}\left(a_{6}-\frac{1}{2} b_{6}\right) & -\frac{\sqrt{3}}{2} b_{7} d_{6}-\frac{\sqrt{3}}{2} b_{6} d_{7} \\
-\frac{2}{\sqrt{3}}\left(a_{7}-\frac{1}{2} b_{7}\right)\left(c_{6}-\frac{1}{2} d_{6}\right)-\frac{2}{\sqrt{3}}\left(a_{6}-\frac{1}{2} b_{6}\right)\left(c_{7}-\frac{1}{2} d_{7}\right) & -d_{6}\left(a_{7}-\frac{1}{2} b_{7}\right)-b_{6}\left(c_{7}-\frac{1}{2} d_{7}\right)
\end{array}\right] .
$$

Thus, $\tilde{U}$ has the shape

$$
\tilde{U}=\left[\begin{array}{cccc}
\frac{1}{2} \tilde{P} & \frac{\sqrt{3}}{2} \tilde{Q} \\
\frac{1}{2 \sqrt{3}} \tilde{R} & \frac{1}{2} & \tilde{S}
\end{array}\right]
$$

where $\tilde{P}, \tilde{Q}, \tilde{R}, \tilde{S} \in \mathbf{Z}$.

As in Case 1, where (3.52) implies (3.53) and thus (3.54), the condition (3.69) implies that $\tilde{U}=\left(\tilde{U}^{-1}\right)^{T}$, so that $\tilde{U}$ is of the form

$$
\tilde{U}=\left[\begin{array}{cc}
\tilde{w} & \tilde{x} \\
-\tilde{x} & \tilde{w}
\end{array}\right]
$$

By (3.70) and (3.71), we see that $\tilde{U}$ is of the form

$$
\tilde{U}=\left[\begin{array}{ccc}
\frac{1}{2} \tilde{P} & \frac{\sqrt{3}}{2} \tilde{Q} \\
-\frac{\sqrt{3}}{2} \tilde{Q} & \frac{1}{2} \tilde{P}
\end{array}\right],
$$

where $\tilde{P}, \tilde{Q} \in \mathbf{Z}$. But note that, by (3.68) and (3.54),

$$
|\tilde{U}|=\left|\tilde{M}_{7}^{-1}\right|\left|M_{6}\right|=\left(-\frac{2}{\sqrt{3}}\right)\left(-\frac{\sqrt{3}}{2}\right)=1,
$$

which implies that

$$
\frac{1}{4} \tilde{P}^{2}+\frac{3}{4} \tilde{Q}^{2}=1
$$


If $\tilde{P}= \pm 2$ and $\tilde{Q}=0$, then, by (3.60) and (3.61), $d_{6}= \pm d_{7}$. If, however, $\tilde{P}$, $\tilde{Q} \in\{ \pm 1\}$, then $\tilde{U}$ has the form

$$
\tilde{U}=\left[\begin{array}{cc} 
\pm \frac{1}{2} & \frac{\sqrt{3}}{2} \\
-\frac{\sqrt{3}}{2} & \pm \frac{1}{2}
\end{array}\right] \quad \text { or } \quad \tilde{U}=\left[\begin{array}{cc} 
\pm \frac{1}{2} & -\frac{\sqrt{3}}{2} \\
\frac{\sqrt{3}}{2} & \pm \frac{1}{2}
\end{array}\right] .
$$

If the first case of (3.73) holds, then, by (3.69),

$$
\left[\begin{array}{cc}
c_{6}-\frac{1}{2} d_{6} & \frac{\sqrt{3}}{2} d_{6} \\
a_{6}-\frac{1}{2} b_{6} & \frac{\sqrt{3}}{2} b_{6}
\end{array}\right]=\left[\begin{array}{cc}
c_{7}-\frac{1}{2} d_{7} & -\frac{\sqrt{3}}{2} d_{7} \\
-\left(a_{7}-\frac{1}{2} b_{7}\right) & \frac{\sqrt{3}}{2} b_{7}
\end{array}\right]\left[\begin{array}{cc} 
\pm \frac{1}{2} & \frac{\sqrt{3}}{2} \\
-\frac{\sqrt{3}}{2} & \pm \frac{1}{2}
\end{array}\right] .
$$

The entry in the first row and second column of the matrix on the left-hand side of the equation is

$$
\frac{\sqrt{3}}{2} d_{6}=\frac{\sqrt{3}}{2}\left(c_{7}-\frac{1}{2} d_{7}\right) \mp \frac{\sqrt{3}}{4} d_{7}=\frac{\sqrt{3}}{2} c_{7}+\left(-\frac{\sqrt{3}}{4} \mp \frac{\sqrt{3}}{4}\right) d_{7},
$$

which implies (3.41), because (3.75) is the same as equation (3.64) which ultimately implied (3.41).

If, however, the second option in (3.73) holds, then by a similar argument,

$$
\frac{\sqrt{3}}{2} d_{6}=-\frac{\sqrt{3}}{2}\left(c_{7}-\frac{1}{2} d_{7}\right) \mp \frac{\sqrt{3}}{4} d_{7}=-\frac{\sqrt{3}}{2} c_{7}+\left(\frac{\sqrt{3}}{4} \mp \frac{\sqrt{3}}{4}\right) d_{7},
$$

which implies (3.41), because (3.76) is the same as equation (3.65) which ultimately implied (3.41).

Thus, (3.41) holds both in Case 1 and Case 2, and the lemma is proved.

\section{The COefFicients of $E_{4} / E_{6}$}

In a letter to Hardy written from Matlock House, an English sanitarium, Ramanujan [19, p. 117] communicated a result which is very similar to formula (3.6) in Theorem 3.1. We describe that result in this section. More details can be found in 1 .

Suppose that $(c, d)$ is a pair of coprime integers which is a solution to the equation

$$
\mu=c^{2}+d^{2},
$$

where $\mu$ is fixed. Let $K=\mathbb{Q}(\sqrt{-1})$. Then $\mathfrak{O}=\mathbb{Z}[\sqrt{-1}]=\mathbb{Z} \oplus \mathbb{Z} i$ is a principal ideal domain. Thus, from 4.1),$\lambda=N(\mathfrak{A})=\mathfrak{A} \overline{\mathfrak{A}}$, where $\mathfrak{A}=(c+d i)=:(\alpha)$ and $\overline{\mathfrak{A}}=(\bar{\alpha})$. The group of units in $\mathfrak{O}_{K}$ is then given by $U=\{ \pm 1, \pm i\}$. Thus,

$$
\begin{aligned}
& \mathfrak{A}=(\alpha)=(-\alpha)=(i \alpha)=(-i \alpha), \\
& \overline{\mathfrak{A}}=(\bar{\alpha})=(-\bar{\alpha})=(i \bar{\alpha})=(-i \bar{\alpha}) .
\end{aligned}
$$

Hence one solution generates a total of eight solutions, namely,

$$
\pm(c, d), \quad \pm(c,-d), \quad \pm(d, c), \quad \pm(d,-c) .
$$

We say that two solutions $\left(c_{1}, d_{1}\right)$ and $\left(c_{2}, d_{2}\right)$ to equation (4.1) are distinct if they do not simultaneously belong to the same set of solutions in (4.2). Note that $\left(c_{1}, d_{1}\right)$ and $\left(c_{2}, d_{2}\right)$ are distinct solutions to (4.1) if and only if

$$
c_{2} \notin\left\{ \pm c_{1}, \pm d_{1}\right\} \text {. }
$$


Recall that [11, p. 164] the integers $\mu$ which can be represented in the form $\mu=c^{2}+d^{2}$, with $c$ and $d$ coprime, are integers of the form

$$
\mu=2^{a} \prod_{j=1}^{r} p_{j}^{a_{j}},
$$

where $a=0$ or $1, p_{j}$ is a prime of the form $4 m+1$, and $a_{j}$ is a nonnegative integer, $1 \leq j \leq r$.

Theorem 4.1. Let

$$
\frac{Q\left(q^{2}\right)}{R\left(q^{2}\right)}=\sum_{n=0}^{\infty} \delta_{n} q^{2 n}
$$

and

$$
J=Q\left(e^{-2 \pi}\right)=1+240 \sum_{k=1}^{\infty} \frac{k^{3}}{e^{2 \pi k}-1}=1.45576 \ldots
$$

Then, if $n \geq 0$,

$$
\begin{aligned}
\delta_{n}= & \frac{2}{J}\left\{e^{2 n \pi}-\frac{(-1)^{n}}{2^{2}} e^{n \pi}+\frac{2 \cos \left(\frac{4 \pi n}{5}+4 \arctan 2\right)}{5^{2}} e^{2 n \pi / 5}\right. \\
& \left.+\frac{2 \cos \left(\frac{3 \pi n}{5}+4 \arctan 3\right)}{10^{2}} e^{2 n \pi / 10}+\cdots\right\} \\
:= & \frac{2}{J} \sum_{(\mu)} \frac{v_{\mu}(n)}{\mu^{2}} e^{2 n \pi / \mu} .
\end{aligned}
$$

Here, $\mu$ runs over the integers of the form (4.4),

$$
v_{1}(n)=1, \quad v_{2}(n)=(-1)^{n+1},
$$

and, for $\mu \geq 5$,

$$
v_{\mu}(n)=2 \sum_{c, d} \cos \left((a c+b d) \frac{2 n \pi}{\mu}+4 \arctan \frac{c}{d}\right),
$$

where the sum is over all pairs $(c, d)$, where $(c, d)$ is a distinct solution to $\mu=c^{2}+d^{2}$ and $(a, b)$ is any solution to $a d-b c=1$. Also, distinct solutions $(c, d)$ to $\mu=c^{2}+d^{2}$ give rise to distinct terms in the sum in (4.7). If $n<0$, then the sum on the far right side of (4.7) equals 0 .

Proof. Let $|q|<1$. We apply Theorem 2.1 to the function $f(q)=Q\left(q^{2}\right) / R\left(q^{2}\right)$. Then $\varphi(\tau)=E_{4}(\tau) / E_{6}(\tau)$. Since $E_{4}(\tau)$ and $E_{6}(\tau)$ are modular forms of degrees -4 and -6 , respectively, $\varphi(\tau)$ satisfies the functional equation (2.1) with $n=2$. The only zero of $E_{6}(\tau)$ in $P_{1}$ is at

$$
\tau=i
$$

(Rankin [20, p. 198]), while by (3.10), $E_{4}(\tau)$ does not have a zero at $\tau=i$. Thus, in the notation of Theorem 2.1.

$$
\alpha=i .
$$


Lastly, because $1 / E_{6}(\tau)$ is meromorphic in the upper half-plane [21, p. 50], we see that $\varphi(\tau)$ is also meromorphic there and that $f(q)$ is meromorphic in the unit circle.

We now calculate $A=\operatorname{Res}(\varphi, i)$. By (3.11), (4.6), and an identity of Ramanujan [17], [18, p. 142, Table II, no. 3],

$$
\begin{aligned}
\operatorname{Res}\left(f, e^{-\pi}\right) & =\left.\frac{Q\left(q^{2}\right)}{d R\left(q^{2}\right) / d q}\right|_{q=e^{-\pi}}=\left.\frac{-q Q\left(q^{2}\right)}{1008 \sum_{k=1}^{\infty} \frac{k^{6} q^{2 k}}{\left(1-q^{2 k}\right)^{2}}}\right|_{q=e^{-\pi}} \\
& =\left.\frac{-q Q\left(q^{2}\right)}{Q^{2}\left(q^{2}\right)-P\left(q^{2}\right) R\left(q^{2}\right)}\right|_{q=e^{-\pi}}=\left.\frac{-q Q\left(q^{2}\right)}{Q^{2}\left(q^{2}\right)}\right|_{q=e^{-\pi}}=\frac{-e^{-\pi}}{J} .
\end{aligned}
$$

Thus, by (4.12) and (2.37),

$$
A=\operatorname{Res}(\varphi, i)=\frac{\operatorname{Res}\left(f, e^{-\pi}\right)}{\pi i e^{-\pi}}=\frac{-1}{J \pi i} .
$$

By (2.2), (4.11), and (4.13), we deduce that

$$
f(q)=\frac{2}{J} \sum_{(c, d)} \frac{1}{(c i+d)^{4}} \frac{1}{1-(q / \underline{q})^{2}},
$$

where

$$
\underline{q}=\exp \left(\pi i\left(\frac{a i+b}{c i+d}\right)\right),
$$

and the conditions on $a, b, c$, and $d$ are the same as in (3.13) and (3.14). We need to explicitly determine the values over which $(c, d)$ runs.

The analysis used to determine which pairs $(c, d)$ are counted in the summation is very similar to that in Theorem 3.1 Now,

$$
\underline{q}=\exp \left(\pi i\left(\frac{(a c+b d)+i}{c^{2}+d^{2}}\right)\right)=\exp \left(\frac{-\pi}{\mu}\right) \exp \left(\frac{\pi i}{\mu}(a c+b d)\right),
$$

where $\mu=c^{2}+d^{2}$. As in (3.16), we can show that if the two pairs $\left(c_{1}, d_{1}\right)$ and $\left(c_{2}, d_{2}\right)$ produce distinct values of $\mu$, then they lead to distinct values for the set $\{ \pm \underline{q}\}$, i.e.,

$$
\left\{ \pm \underline{q}_{1}\right\} \wedge\left\{ \pm \underline{q}_{2}\right\}=\emptyset .
$$

When $\mu=1$ or $\mu=2$, then only four of the eight pairs in (4.2) are distinct. Each value of $\mu$ corresponds to only two values of $\underline{q}$, say, $\left\{ \pm \underline{q}_{3}\right\}$. When $\mu \geq 5$, all eight pairs in (4.2) are distinct. However, if $(c, d)$ leads to $\left\{ \pm \underline{q}_{4}\right\}$, say, then $(-c, d)$ and $(d, c)$ each lead to $\left\{ \pm \underline{q}_{4}\right\}$. It follows that the eight pairs of solutions in (4.2) lead to only four different poles, namely,

$$
\left\{ \pm q_{4}, \pm \underline{q}_{4}\right\} .
$$

These four poles are indeed distinct, because, as we will show, in Lemmas 4.24 .4 at the end of the section, $\underline{q}$ is real only for $\mu=1$, and $\underline{q}$ is purely imaginary only for $\mu=2$. 
Lastly, in Lemma 4.5. we prove that if $\mu \geq 5$ and $\left(c_{1}, d_{1}\right)$ and $\left(c_{2}, d_{2}\right)$, say, are distinct solutions to equation (4.1), then each solution, taken together with the seven corresponding solutions in (4.2), yields four distinct poles, i.e.,

$$
\left\{ \pm \underline{q}_{1}, \pm \underline{\bar{q}}_{1}\right\} \wedge\left\{ \pm \underline{q}_{2}, \pm \underline{q}_{2}\right\}=\emptyset
$$

In summary, we have so far shown, by (4.14) and (4.17)-(4.19), that

$$
\begin{aligned}
f(q)= & \frac{2}{J}\left\{\sum_{\substack{(\mu) \\
\mu \leq 2}} \frac{1}{(c i+d)^{4}} \frac{1}{1-(q / \underline{q})^{2}}\right. \\
& \left.+\sum_{\substack{(\mu) \\
\mu>2}}\left(\frac{1}{(c i+d)^{4}} \frac{1}{1-(q / \underline{q})^{2}}+\frac{1}{(-c i+d)^{4}} \frac{1}{1-(q / \underline{\bar{q}})^{2}}\right)\right\},
\end{aligned}
$$

where $\mu$ runs over all integers of the form (4.4), and where, for each fixed $\mu$, the sum is also over all distinct pairs $(c, d)$.

For $\mu=1$ and, say, $(a, b, c, d)=(1,0,0,1)$, we find that, by (4.16), $\underline{q}=e^{-\pi}$, so that the summand in (4.20) is

$$
\frac{1}{1-q^{2} e^{2 \pi}}
$$

For $\mu=2$ and, say, $(a, b, c, d)=(1,0,1,1)$, we find that $\underline{q}=i e^{-\pi / 2}$, so that the summand in (4.20) is

$$
-\frac{1}{2^{2}} \frac{1}{1+q^{2} e^{\pi}}
$$

Thus, by (4.21) and (4.22), we can rewrite (4.20) as

$$
\begin{aligned}
f(q)= & \frac{2}{J}\left\{\frac{1}{1-q^{2} e^{2 \pi}}-\frac{1}{2^{2}} \frac{1}{1+q^{2} e^{\pi}}\right. \\
& \left.+\sum_{(\mu)}\left(\frac{1}{(c i+d)^{4}} \frac{1}{1-(q / \underline{q})^{2}}+\frac{1}{(-c i+d)^{4}} \frac{1}{1-(q / \underline{\bar{q}})^{2}}\right)\right\} \\
= & \frac{2}{J}\left\{\sum_{n=0}^{\infty} e^{2 n \pi} q^{2 n}-\frac{1}{2^{2}} \sum_{n=0}^{\infty}(-1)^{n} e^{n \pi} q^{2 n}\right. \\
& \left.+\sum_{(\mu)}\left(\frac{1}{(c i+d)^{4}} \sum_{n=0}^{\infty} \underline{q}^{-2 n} q^{2 n}+\frac{1}{(-c i+d)^{4}} \sum_{n=0}^{\infty} \underline{\bar{q}}^{-2 n} q^{2 n}\right)\right\} \\
= & \sum_{n=0}^{\infty} \delta_{n} q^{2 n},
\end{aligned}
$$


where $|q|<e^{-\pi}$ and

$$
\begin{aligned}
\delta_{n} & =\frac{2}{J}\left\{e^{2 n \pi}-\frac{(-1)^{n}}{2^{2}} e^{n \pi}+\sum_{\substack{(\mu) \\
\mu>2}}\left(\frac{1}{(c i+d)^{4}} \underline{q}^{-2 n}+\frac{1}{(-c i+d)^{4}} \bar{q}^{-2 n}\right)\right\} \\
& =\frac{2}{J}\left\{e^{2 n \pi}-\frac{(-1)^{n}}{2^{2}} e^{n \pi}+\sum_{\substack{\mu \\
\mu>2}} \frac{2 \cos \left((a c+b d) \frac{2 n \pi}{\mu}+4 \arctan \frac{c}{d}\right)}{\mu^{2}} e^{2 n \pi / \mu}\right\} \\
(4.24) & =\frac{2}{J} \sum_{(\mu)} \frac{v_{\mu}(n) e^{2 n \pi / \mu}}{\mu^{2}},
\end{aligned}
$$

where $v_{\mu}(n)$ is defined in (4.8) and (4.9). To obtain the displayed terms in (4.7), we choose $(a, b, c, d)=(1,0,2,1)$ for $\mu=5$ and $(a, b, c, d)=(1,0,3,1)$ for $\mu=10$.

Thus, apart from the lemmas below, the proof of Theorem 4.1 is complete for $n \geq 0$. For $n<0$, we repeat the argument above but with $|q|>1$. Then, by Theorem 2.1 the left side of (4.23) equals 0 instead of $f(q)$. We now expand the series on the left side of (4.23) in powers of $1 / q$ instead of powers of $q$. We complete the argument as in the proof of Theorem 3.1

The following four lemmas are analogous to Lemmas 3.2 3.5, and their proofs are very similar. We forego the proofs, since they are similar to those in the previous section.

Lemma 4.2. Given a pair of coprime integers $(c, d)$, we can always choose integers $a$ and $b$ such that $a d-b c=1$ and

$$
|a c+b d| \leq \frac{1}{2}\left(c^{2}+d^{2}\right) .
$$

Lemma 4.3. If $a d-b c=1$, where $a, b, c, d \in \mathbf{Z}$, then the quantity

$$
\underline{q}=\exp \left(\frac{-\pi}{c^{2}+d^{2}}\right) \exp \left(\frac{\pi i}{c^{2}+d^{2}}(a c+b d)\right)
$$

is real only when $c^{2}+d^{2}=1$.

Lemma 4.4. The quantity $\underline{q}$ is imaginary only when $c^{2}+d^{2}=2$.

Lemma 4.5. If $\left(c_{1}, d_{1}\right)$ and $\left(c_{2}, d_{2}\right)$, say, are two pairs of coprime integers such that

$$
c_{1}^{2}+d_{1}^{2}=c_{2}^{2}+d_{2}^{2}=\mu,
$$

and if

$$
\left\{ \pm \underline{q}_{1}, \pm \underline{\bar{q}}_{1}\right\}=\left\{ \pm \underline{q}_{2}, \pm \underline{\bar{q}}_{2}\right\}
$$

where $q$ is defined in (4.16), then the two solutions of (4.27) are not distinct. In other words,

$$
c_{2} \in\left\{ \pm c_{1}, \pm d_{1}\right\} .
$$




\section{The COEFFiCients of $\left(\pi E_{2}^{*} / 3\right) / E_{6}$ AND $\left(\pi E_{2}^{*} / 3\right)^{2} / E_{6}$}

The following theorem is from the fragment published with the "lost notebook" [19, pp. 102-104] and is similar to the previous theorems. However, since this result involves a function which is not a modular form, we need to modify Theorem 2.1 in order to prove the result.

Theorem 5.1. Let

$$
\frac{\frac{\pi}{3} P\left(q^{2}\right)}{R\left(q^{2}\right)}=\sum_{n=0}^{\infty} \eta_{1, n} q^{2 n}
$$

and

$$
\frac{\left(\frac{\pi}{3} P\left(q^{2}\right)\right)^{2}}{R\left(q^{2}\right)}=\sum_{n=0}^{\infty} \eta_{2, n} q^{2 n} .
$$

If

$$
C:=1+480 \sum_{k=1}^{\infty} \frac{k^{7}}{e^{2 \pi k}-1}
$$

then, if $n \geq 0$,

$$
\begin{aligned}
\eta_{1, n}= & \frac{2}{C}\left\{e^{2 n \pi}-\frac{(-1)^{n}}{2^{3}} e^{n \pi}+\frac{2 \cos \left(\frac{4 \pi n}{5}+8 \arctan 2\right)}{5^{3}} e^{2 n \pi / 5}\right. \\
& \left.+\frac{2 \cos \left(\frac{3 \pi n}{5}+8 \arctan 3\right)}{10^{3}} e^{2 n \pi / 10}+\cdots\right\} \\
= & : \frac{2}{C} \sum_{(\mu)} \frac{W_{\mu}(n)}{\mu^{3}} e^{2 n \pi / \mu}
\end{aligned}
$$

and

$$
\eta_{2, n}=\frac{2}{C} \sum_{(\mu)} \frac{W_{\mu}(n)}{\mu^{2}} e^{2 n \pi / \mu},
$$

where $\mu$ runs over the integers of the form (4.4). Here,

$$
W_{1}(n)=1, \quad W_{2}(n)=-(-1)^{n},
$$

and, for $\mu \geq 5$,

$$
W_{\mu}(n)=2 \sum_{c, d} \cos \left((a c+b d) \frac{2 n \pi}{\mu}+8 \arctan \frac{c}{d}\right),
$$

where the sum is over all pairs $(c, d)$, where $(c, d)$ is a distinct solution of $\mu=c^{2}+d^{2}$. Also, distinct solutions $(c, d)$ to $\mu=c^{2}+d^{2}$ give rise to distinct terms in the sums in (5.4) and (5.5). If $n<0$, then the sums on the right sides of (5.4) and (5.5) are both equal to 0 . 
Note that the definition of $W_{\mu}(n)$ is almost identical to that of $v_{\mu}(n)$ in Theorem 4.1 .

Proof. For $j=1$ or 2 , let $f_{j}(q)$ denote the quotients on the left sides of (5.1) and (5.2), respectively. Define $\varphi_{j}(\tau)=f_{j}\left(e^{\pi i \tau}\right)$. Then, by (1.5) and (1.4),

$$
\varphi_{1}(\tau)=\frac{\pi E_{2}^{*}(\tau) / 3}{E_{6}(\tau)} \quad \text { and } \quad \varphi_{2}(\tau)=\frac{\left(\pi E_{2}^{*}(\tau) / 3\right)^{2}}{E_{6}(\tau)} .
$$

Recall that $E_{2}(\tau)$, defined by (1.6), satisfies the functional equation

$$
E_{2}(V \tau)=E_{2}(\tau)(c \tau+d)^{2}
$$

for any modular transformation $V \tau=(a \tau+b) /(c \tau+d)$. Although $E_{2}^{*}(\tau)$ is not a modular form, we see from Ramanujan's work [3, p. 320] that it does satisfy a modified functional equation:

$$
E_{2}^{*}(V \tau)=E_{2}^{*}(\tau)(c \tau+d)^{2}-\frac{6 c i}{\pi}(c \tau+d) .
$$

Since $E_{6}$ is a modular form of weight 6 ,

$$
E_{6}(V \tau)=E_{6}(\tau)(c \tau+d)^{6} .
$$

Taking (5.10) and (5.11) together, we find that

$$
\varphi_{1}(\tau)=\varphi_{1}(V \tau)(c \tau+d)^{4}+\frac{2 c i}{E_{6}(V \tau)}(c \tau+d)^{5}
$$

and, after squaring both sides of (5.10),

$$
\varphi_{2}(\tau)=\varphi_{2}(V \tau)(c \tau+d)^{2}+4 c i \varphi_{1}(V \tau)(c \tau+d)^{3}-\frac{4 c^{2}}{E_{6}(V \tau)}(c \tau+d)^{4} .
$$

We now prove that modified versions of Theorem 2.1 hold when the functional equation (2.1) is replaced by either (5.12) or (5.13).

When we replace (2.1) by either (5.12) or (5.13), the parts of the proof which are affected are the estimation of the integral (2.5) and the calculation of the residues of $\varphi_{j}$. The only resulting change in the statement of the theorem itself is a slight modification of (2.2).

When we replace (2.1) by (5.12) or (5.13), the function $\varphi_{j}(\tau)$ satisfies the conditions of Theorem 2.1 for $j=1$ or 2 with $\alpha=i$, because $P(q)$ is analytic in the unit circle.

We now estimate the integral (2.5). Let $V^{*} \tau=\left(k^{\prime} \tau-h^{\prime}\right) /(-k \tau+h)$. By (5.12) and (5.13), our estimates for $\varphi$ in (2.24) are replaced by, respectively,

$$
\left|\varphi_{1}(\tau)\right|<\left|\varphi_{1}\left(V^{*} \tau\right)\right||-k \tau+h|^{4}+\frac{2 k}{\left|E_{6}\left(V^{*} \tau\right)\right|}|-k \tau+h|^{5}
$$

and

$$
\begin{aligned}
\left|\varphi_{2}(\tau)\right|< & \left|\varphi_{2}\left(V^{*} \tau\right)\right||-k \tau+h|^{2}+4 k\left|\varphi_{1}\left(V^{*} \tau\right)\right||-k \tau+h|^{3} \\
& +\frac{4 k^{2}}{\left|E_{6}\left(V^{*} \tau\right)\right|}|-k \tau+h|^{4} .
\end{aligned}
$$


As we have seen, Theorem 2.1 can be applied to the function $1 / E_{6}(\tau)$ (as Hardy and Ramanujan [10] did). Thus, on the boundary of $P$,

$$
\left|\frac{1}{E_{6}(\tau)}\right|<\tilde{M}
$$

for some positive constant $\tilde{M}$. By (5.14), (5.16), (2.22), and (2.25),

$$
\begin{aligned}
\left|\varphi_{1}(\tau)\right| & <M|-k \tau+h|^{4}+2 k \tilde{M}|-k \tau+h|^{5} \\
& =k^{4} M|\tau-h / k|^{4}+2 k^{6} \tilde{M}|\tau-h / k|^{5} \\
& <\frac{16 M}{\left(k^{2}+k k^{\prime}+k^{\prime 2}\right)^{2}}+\frac{64 k \tilde{M}}{\left(k^{2}+k k^{\prime}+k^{\prime 2}\right)^{5 / 2}} \\
& <\frac{M_{0}}{\left(k^{2}+k k^{\prime}+k^{\prime 2}\right)^{2}},
\end{aligned}
$$

where $M_{0}$ is some positive constant.

Similarly,

$$
\left|\varphi_{2}(\tau)\right|<\frac{\hat{M}_{0}}{k^{2}+k k^{\prime}+k^{\prime 2}},
$$

where $\hat{M}_{0}$ is some positive constant. Note that the inequalities (5.17) and (5.18) are similar to (2.26). The remainder of the argument is the same as before, and so the integral in (2.5) approaches 0 as $m$ approaches $\infty$ in the cases of $\varphi_{1}$ and $\varphi_{2}$.

Next we evaluate $\operatorname{Res}\left(\varphi_{j}, i\right)$ using the following lemma.

Lemma 5.2. Let $V \tau=(a \tau+b) /(c \tau+d)$, where $a, b, c$, and $d$ are integers satisfying $a d-b c=1$. Then

$$
\frac{\pi}{3} E_{2}^{*}(V i)=\frac{\pi}{3} P\left(\underline{q}^{2}\right)=c^{2}+d^{2},
$$

where

$$
\underline{q}=\exp \left(\pi i\left(\frac{a i+b}{c i+d}\right)\right) .
$$

Proof. Consider the modular form $E_{2}(\tau)$ defined in (1.6). From [2, p. 159] and [3. p. 256],

$$
E_{2}(i)=1-24 \sum_{k=1}^{\infty} \frac{k}{e^{2 \pi k}-1}-\frac{3}{\pi}=0 .
$$

By (5.20), (1.6), and the definition of $\underline{q}$ above,

$$
\begin{aligned}
0 & =E_{2}(V i)=1-24 \sum_{k=1}^{\infty} \frac{k \underline{q}^{2 k}}{1-\underline{q}^{2 k}}-\frac{3}{\pi \operatorname{Im}(V i)} \\
& =1-24 \sum_{k=1}^{\infty} \frac{k \underline{q}^{2 k}}{1-\underline{q}^{2 k}}-\frac{3}{\pi}\left(c^{2}+d^{2}\right),
\end{aligned}
$$

and hence we obtain (5.19).

By (2.36), or more precisely the sentence preceding (2.36), and (4.13),

$$
\operatorname{Res}\left(\frac{1}{E_{6}(\tau)}, V i\right)=\frac{\operatorname{Res}\left(1 / E_{6}(\tau), i\right)}{(c i+d)^{8}}=\frac{-1 /\left(J^{2} \pi i\right)}{(c i+d)^{8}}=\frac{-1 /(C \pi i)}{(c i+d)^{8}}
$$


by a result of Ramanujan [17, Table I, Entry 4], [18, p. 141], where $J$ is defined in (4.6) and $C$ is defined in (5.3). By (2.37) and the calculation above,

$$
\operatorname{Res}\left(\frac{1}{R\left(q^{2}\right)}, \underline{q}\right)=-\frac{\underline{q}}{C(c i+d)^{8}} \text {. }
$$

It follows from (5.19) and (5.21) that

$$
\operatorname{Res}\left(f_{j}(q), \underline{q}\right)=-\left(c^{2}+d^{2}\right)^{j} \frac{\underline{q}}{C(c i+d)^{8}} .
$$

When we replace (2.37) with (5.22), the analogue of (2.38) becomes, or, alternatively, (2.33) becomes

$$
f_{j}(q)=\frac{2}{C} \sum_{(c, d)} \frac{\mu^{j}}{(c i+d)^{8}} \frac{1}{1-(q / \underline{q})^{2}},
$$

where $\mu=c^{2}+d^{2}$.

Thus, the conclusion of Theorem 2.1 is valid for $\varphi_{j}$ if we replace (2.2) by (5.23).

Since the quantity $q$ is the same for $\varphi_{j}$ as for $\varphi$ in Theorem 4.1, the analysis involving the values of $(c, d)$ counted in the summation in (4.14) is valid for $\varphi_{j}$ as well. So by (4.20), (4.23), and (4.24), we find that

$$
f_{j}(q)=\sum_{n=0}^{\infty} \eta_{j, n} q^{2 n}
$$

where, for $j=1,2$,

$$
\begin{aligned}
\eta_{j, n} & =\frac{2}{C}\left\{e^{2 n \pi}-\frac{(-1)^{n}}{2^{4-j}} e^{n \pi}+\sum_{\substack{(\mu) \\
\mu>2}} \frac{2 \cos \left((a c+b d) \frac{2 n \pi}{\mu}+8 \arctan \frac{d}{c}\right)}{\mu^{4-j}} e^{2 n \pi / \mu}\right\} \\
(5.25) & =\frac{2}{C} \sum_{(\mu)} \frac{W_{\mu}(n) e^{2 n \pi / \mu}}{\mu^{4-j}},
\end{aligned}
$$

by (5.7). In (5.24), as in (4.20), $\mu$ runs over all integers of the form (4.4), and for each fixed $\mu$, the sum is also over all distinct pairs $(c, d)$.

Thus, the proof of (5.4) is complete.

\section{The COefFicients of $\left(\pi E_{2}^{*} / 2 \sqrt{3}\right) / E_{4}$}

The theorem in this section is from the same fragment [19, pp. 102-104] as the previous theorem, and the proof is very similar, and so we forego it. More details may be found in [1].

Theorem 6.1. Let

$$
f(q):=\frac{\pi P\left(q^{2}\right)}{2 \sqrt{3} Q\left(q^{2}\right)}=\sum_{n=0}^{\infty} \theta_{n} q^{2 n} .
$$


Then, if $n \geq 0$,

$$
\theta_{n}=(-1)^{n} \frac{3}{G} \sum_{(\lambda)} \frac{h_{\lambda}(n)}{\lambda^{2}} e^{n \pi \sqrt{3} / \lambda},
$$

where $\lambda$ runs over the integers of the form (3.4), and $G$ and $h_{\lambda}(n)$ are defined in (3.5) and (3.8), respectively. Also, distinct solutions $(c, d)$ to $\lambda=c^{2}-c d+d^{2}$, which were defined before Theorem [3.1, give rise to distinct terms in the sum in (6.2). If $n<0$, the sum on the right side of (6.2) equals 0 .

We are grateful to R. J. Evans, L.-C. Zhang, and the referee for very helpful suggestions.

\section{REFERENCES}

[1] G. E. Andrews and B. C. Berndt, Ramanujan's Lost Notebook, Part II, Springer, New York, to appear.

[2] B. C. Berndt, Modular transformations and generalizations of several formulae of Ramanujan, Rocky Mt. J. Math. 7 (1977), 147-189. MR0429703 (55:2714)

[3] B. C. Berndt, Ramanujan's Notebooks, Part II, Springer-Verlag, New York, 1989. MR0970033 (90b:01039)

[4] B. C. Berndt, Ramanujan's Notebooks, Part III, Springer-Verlag, New York, 1991. MR.1117903 (92j:01069)

[5] B. C. Berndt, P. R. Bialek, and A. J. Yee, Formulas of Ramanujan for the power series coefficients of certain quotients of Eisenstein series, International Mathematics Research Notices 2002, no. 21, 1077-1109. MR1904462 (2003j:11047)

[6] B. C. Berndt and R. A. Rankin, Ramanujan: Letters and Commentary, History of Math., vol. 9, American Mathematical Society, Providence, 1995; London Mathematical Society, London, 1995. MR.1353909 (97c:01034)

[7] P. R. Bialek, Ramanujan's Formulas for the Coefficients in the Power Series Expansions of Certain Modular Forms, Ph.D. thesis, University of Illinois at Urbana-Champaign, Urbana, IL, 1995.

[8] G. H. Hardy and E. M. Wright, An Introduction to the Theory of Numbers, 4th ed., Clarendon Press, Oxford, 1960.

[9] G. H. Hardy and S. Ramanujan, Asymptotic formulae in combinatory analysis, Proc. London Math. Soc. (2) 17 (1918), 75-118.

[10] G. H. Hardy and S. Ramanujan, On the coefficients in the expansions of certain modular functions, Proc. Royal Soc. A 95 (1918), 144-155.

[11] I. Niven, H. S. Zuckerman, and H. L. Montgomery, An Introduction to the Theory of Numbers, 5th ed., Wiley, New York, 1991. MR1083765 (91i:11001)

[12] J. Lehner, The Fourier coefficients of automorphic forms on horocyclic groups, III, Mich. Math. J. 7 (1960), 65-74. MR0126550|(23:A3846)

[13] H. Petersson, Konstruktion der Modulformen und der zu gewissen Grenzkreisgruppen gehörigen automorphen Formen von positiver reeller Dimension und die vollständige Bestimmung ihrer Fourierkoeffzienten, S.-B. Heidelberger Akad. Wiss. Math. Nat. Kl. (1950), 415-494. MR0041172 (12:806e)

[14] H. Petersson, Uber automorphe Orthogonalfunktionen und die Konstruktion der automorphen Formen von positiver reeller Dimension, Math. Ann. 127 (1954), 33-81. MR 0060542 $(15: 686 \mathrm{e})$

[15] H. Petersson, Über automorphe Formen mit Singularitäten im Diskontinuitätsgebiet, Math. Ann. 129 (1955), 370-390. MR0071459 (17:129c)

[16] H. Poincaré, Oeuvres, Vol. 2, Gauthiers-Villars, Paris, 1916.

[17] S. Ramanujan, On certain arithmetical functions, Trans. Cambridge Philos. Soc. 22 (1916), $159-184$

[18] S. Ramanujan, Collected Papers, Cambridge University Press, Cambridge, 1927; reprinted by Chelsea, New York, 1962; reprinted by the American Mathematical Society, Providence, RI, 2000. 
[19] S. Ramanujan, The Lost Notebook and Other Unpublished Papers, Narosa, New Delhi, 1988. MR0947735 (89j:01078)

[20] R. A. Rankin, Modular Forms and Functions, Cambridge University Press, Cambridge, 1977. MR 0498390 (58:16518)

[21] B. Schoeneberg, Elliptic Modular Functions, Springer-Verlag, New York, 1974. MR0412107 $(54: 236)$

[22] H. S. Zuckerman, On the expansion of certain modular forms of positive dimension, Amer. J. Math. 62 (1940), 127-152. MF.0001306 (1:214c)

Department of Mathematics, University of Illinois, 1409 West Green Street, UrBANA, ILLINOIS 61801

E-mail address: berndt@math.uiuc.edu

Department of Mathematics, Trinity International University, 2065 Half Day Road, DeERFIEld, ILLinois 60015

E-mail address: pbialek@trin.edu 